\title{
RELATIVE DONALDSON-THOMAS THEORY FOR CALABI-YAU 4-FOLDS
}

\author{
YALONG CAO AND NAICHUNG CONAN LEUNG
}

\begin{abstract}
Given a complex 4-fold $X$ with an (Calabi-Yau 3-fold) anticanonical divisor $Y$, we study relative Donaldson-Thomas invariants for this pair, which are elements in the Donaldson-Thomas cohomologies of $Y$. We also discuss gluing formulas which relate relative invariants and $D T_{4}$ invariants for Calabi-Yau 4-folds.
\end{abstract}

\section{INTRODUCTION}

Donaldson-Thomas invariants ( $D T_{3}$ invariants for short) were proposed by Donaldson and Thomas [19] and defined in Thomas' thesis [54. They count stable sheaves on Calabi-Yau 3-folds, which are related to many other interesting subjects, including the Gopakumar-Vafa conjecture on BPS numbers in string theory [24], 26], 33] and the Maulik-Nekrasov-Okounkov-Pandharipande (MNOP) conjecture 43, 44, 45, 50, relating $D T_{3}$ invariants to Gromov-Witten invariants. The generalization of $D T_{3}$ invariants to count strictly semi-stable sheaves is due to Joyce and Song [32] using Behrend's result [4].

Kontsevich and Soibelman proposed generalized as well as motivic DT theory for Calabi-Yau 3-categories [34, which was later studied by Behrend-Bryan-Szendröi [5] for Hilbert schemes of points. The wall-crossing formula [34, 32] is an important structure for Bridgeland's stability condition [10] and Pandharipande-Thomas invariants [51, 55].

As a categorification of Donaldson-Thomas invariants, Brav, Bussi, Dupont, Joyce and Szendroi [8] and Kiem and Li 33 recently defined a cohomology theory for Calabi-Yau 3-folds whose Euler characteristic is the $D T_{3}$ invariant. The point is that moduli spaces of simple sheaves on Calabi-Yau 3-folds are critical points of holomorphic functions locally [9], 32, and we could consider perverse sheaves of vanishing cycles of these functions. They glued these local perverse sheaves and defined its hypercohomology as $D T_{3}$ cohomology. In general, such a gluing requires a square root of the determinant line bundle of the moduli space [27, 34, 49].

As an extension of Donaldson-Thomas invariants to Calabi-Yau 4-folds, Borisov and Joyce [7] and the authors [12, 13] developed $D T_{4}$ invariants (or 'holomorphic Donaldson invariants') which count stable sheaves on Calabi-Yau 4-folds. It is desirable to construct a TQFT type structure for these $D T_{4}$ and $D T_{3}$ theories. The purpose of this paper is to make some initial steps in this direction. We remark

Received by the editors November 4, 2015 and, in revised form, June 14, 2016.

2010 Mathematics Subject Classification. Primary 14N35; Secondary 14J32.

The second author was supported by grants from the Research Grants Council of the Hong Kong Special Administrative Region, China (Project Nos. CUHK401411 and CUHK14302714). 
that Joyce also has a program of establishing TQFT structures on Calabi-Yau 3and 4-folds 31] using Pantev-Töen-Vaquié-Vezzosi's shifted symplectic structures on derived schemes 52 .

Our set-up is a smooth Calabi-Yau 3-fold $Y=s^{-1}(0)$ as an anti-canonical divisor of a complex projective 4 -fold $X$, where $s \in \Gamma\left(X, K_{X}^{-1}\right)$. Then $1 / s$ is nowhere vanishing inside $X \backslash Y$ which gives a trivialization of its canonical bundle. Thus $X \backslash Y$ is an open Calabi-Yau 4-fold which has a compactification $X$ by adding a compact Calabi-Yau 3-fold.

We consider any Gieseker moduli space $\mathfrak{M}_{X}$ of semi-stable sheaves which consists of slope-stable bundles only, and assume there exists a restriction morphism $r$ : $\mathfrak{M}_{X} \rightarrow \mathfrak{M}_{Y}$ to a Gieseker moduli space of stable sheaves on $Y$ (see Theorem 3.1 for its existence). The deformation-obstruction theory associated to $r$ is described as follows: for any stable bundle $E \in \mathfrak{M}_{X}$, we have an exact sequence

$$
\begin{aligned}
& 0 \rightarrow H^{1}\left(X, \operatorname{End}_{0} E \otimes K_{X}\right) \rightarrow H^{1}\left(X, \operatorname{End}_{0} E\right) \rightarrow H^{1}\left(Y,\left.\operatorname{End}_{0} E\right|_{Y}\right) \\
& \rightarrow H^{2}\left(X, \operatorname{End}_{0} E \otimes K_{X}\right) \rightarrow H^{2}\left(X, E n d_{0} E\right) \rightarrow H^{2}\left(Y,\left.E_{0} E\right|_{Y}\right) \\
& \rightarrow H^{3}\left(X, E n d_{0} E \otimes K_{X}\right) \rightarrow H^{3}\left(X, E n d_{0} E\right) \rightarrow 0 .
\end{aligned}
$$

Note that the transpose of the above sequence with respect to Serre duality pairings on $X$ and $Y$ is itself [19. This is the key property for the definition of relative $D T_{4}$ virtual cycles, which we define for the following three good cases.

Case I (Rigid case). If every $E \in \mathfrak{M}_{X}$ satisfies $H^{1}\left(Y,\left.E n d_{0} E\right|_{Y}\right)=0$, then the above long exact sequence breaks into canonical isomorphisms

$$
H^{1}\left(X, E_{n d} E\right) \cong H^{3}\left(X, E_{n} d_{0} E\right)^{*}, \quad H^{2}\left(X, E_{n} d_{0} E\right) \cong H^{2}\left(X, E_{n} d_{0} E\right)^{*} .
$$

Similar to the case of Calabi-Yau 4-folds [7, $\mathfrak{M}_{X}$ will have a (-2)-shifted symplectic structure in the sense of PTVV [52]. By Borisov-Joyce [7], there exists a virtual cycle, which we define to be the relative $D T_{4}$ virtual cycle.

Case II (Surjective case). If $r: \mathfrak{M}_{X} \rightarrow \mathfrak{M}_{Y}$ is a surjective map between smooth moduli spaces (throughout this paper, unless specified otherwise, smooth moduli spaces mean their Kuranishi maps are zero), we obtain a canonical isomorphism

$$
H^{2}\left(X, \operatorname{End}_{0} E\right) \cong H^{2}\left(X, \operatorname{End}_{0} E\right)^{*},
$$

which endows the obstruction bundle $O b_{\mathfrak{M}_{X}}$ with a non-degenerate quadratic form. Then the relative $D T_{4}$ virtual cycle is defined to be the Euler class of the self-dual subbundle of $O b_{\mathfrak{M}_{X}}$ as in Definition 5.12 [13.

Case III (Injective case). If $r: \mathfrak{M}_{X} \rightarrow \mathfrak{M}_{Y}$ is an injective map between smooth moduli spaces, we obtain an exact sequence

$$
\begin{aligned}
& 0 \rightarrow H^{1}\left(X, E_{n d} E\right) \rightarrow H^{1}\left(Y,\left.E_{n} d_{0} E\right|_{Y}\right) \rightarrow H^{2}\left(X, E_{n} d_{0} E \otimes K_{X}\right) \\
\rightarrow & H^{2}\left(X, E_{0} E\right) \rightarrow H^{2}\left(Y,\left.E n d_{0} E\right|_{Y}\right) \rightarrow H^{3}\left(X, E_{0} E \otimes K_{X}\right) \rightarrow 0 .
\end{aligned}
$$

This determines a surjective map

$$
s: O b_{\mathfrak{M}_{X}} \rightarrow \mathcal{N}_{\mathfrak{M}_{X} / \mathfrak{M}_{Y}}^{*}
$$

from the obstruction bundle of $\mathfrak{M}_{X}$ to the conormal bundle of $\mathfrak{M}_{X}$ inside $\mathfrak{M}_{Y}$, and a non-degenerate quadratic form on the reduced bundle $O b_{\mathfrak{M}_{X}}^{\text {red }} \triangleq \operatorname{Ker}(s)$. As in Case II, we define the relative $D T_{4}$ virtual cycle $\left[\mathfrak{M}_{X}^{r e l}\right]^{\text {vir }} \in H_{*}\left(\mathfrak{M}_{X}, \mathbb{Z}\right)$ to be the 
Euler class of the self-dual subbundle of $O b_{\mathfrak{M}_{X}}^{r e d}$. Note that when $\mathfrak{M}_{X}$ is smooth, $r$ is injective and a neighbourhood of $r\left(\mathfrak{M}_{X}\right) \subseteq \mathfrak{M}_{Y}$ is smooth; [ $\left.\mathfrak{M}_{X}^{\text {rel }}\right]^{\text {vir }}$ can also be defined in a similar way. It is easy to check that these definitions of relative $D T_{4}$ virtual cycles in Cases I-III are all compatible. We compute examples for relative $D T_{4}$ virtual cycles in Proposition 3.10.

To sum up, we define the notion of admissibility for Gieseker moduli spaces.

Definition 1.1. Let $Y$ be a smooth anti-canonical divisor of a projective 4-fold $X$, and $\mathfrak{M}_{X}$ be a Gieseker moduli space of semi-stable sheaves. $\mathfrak{M}_{X}$ is admissible with respect to $(X, Y)$ if

(i) $\mathfrak{M}_{X}$ consists of slope-stable bundles only, and

(ii) there exists a restriction morphism $r: \mathfrak{M}_{X} \rightarrow \mathfrak{M}_{Y}$ to a Gieseker moduli space of stable sheaves on $Y 1$

Theorem/Definition 1.2. If $\mathfrak{M}_{X}$ is admissible with respect to $(X, Y)$, then the relative $\mathrm{DT}_{4}$ virtual cycle exists, i.e.

$$
\left[\mathfrak{M}_{X}^{r e l}\right]^{\text {vir }} \in H_{*}\left(\mathfrak{M}_{X}, \mathbb{Z}_{2}\right),
$$

provided that any one of the following conditions holds:

(1) $r\left(\mathfrak{M}_{X}\right)$ is rigid, i.e. $H^{1}\left(Y,\left.E n d_{0} E\right|_{Y}\right)=0$ for any $E \in \mathfrak{M}_{X}$; or

(2) $r$ is surjective between smooth moduli spaces; or

(3) $r$ is injective between smooth moduli spaces (at least when restricted to a neighbourhood of $r\left(\mathfrak{M}_{X}\right)$ in $\left.\mathfrak{M}_{Y}\right)$.

Furthermore, $\left[\mathfrak{M}_{X}^{r e l}\right]^{\text {vir }}$ will be defined over integers if $r: \mathfrak{M}_{X} \rightarrow \mathfrak{M}_{Y}$ has a relative orientation (Definition 1.7$) 2$

In general, the virtual dimension of $\left[\mathfrak{M}_{X}^{r e l}\right]^{v i r}$ is not zero, and we introduce the $\mu$-map to cut it down and define the relative $D T_{4}$ invariant. The relative $D T_{4}$ invariant is a map

$$
v\left(\mathfrak{M}_{X}\right): \operatorname{Sym}^{*}\left(H_{*}(X, \mathbb{Z}) \otimes \mathbb{Z}\left[x_{1}, x_{2}, \ldots\right]\right) \rightarrow \mathbb{H}^{*}\left(\mathfrak{M}_{Y}, \mathcal{P}_{\mathfrak{M}_{Y}}^{\bullet}\right),
$$

where $\mathcal{P}_{\mathfrak{M}_{Y}}^{\bullet}$ is the perverse sheaf constructed by Brav-Bussi-Dupont-Joyce-Szendroi [8] and Kiem-Li [33. In Cases I-III, if $\mathfrak{M}_{Y}$ is smooth with respect to the natural orientation $K_{\mathfrak{M}_{Y}} 3$ the perverse sheaf $\mathcal{P}_{\mathfrak{M}_{Y}}$ is the $\mathbb{C}$-constant sheaf (up to some degree shift), and the relative $D T_{4}$ invariant $v\left(\mathfrak{M}_{X}\right)$ is defined by pairing the relative $D T_{4}$ virtual cycle, $\mu$-map and pull-back classes from $H^{*}\left(\mathfrak{M}_{Y}, \mathbb{C}\right)$.

So far, we have only worked with holomorphic bundles (i.e. $\mathfrak{M}_{X}$ consists of bundles only). To extend to other coherent sheaves, say ideal sheaves of subschemes, one difficulty is that we will not have well-defined restriction maps in the usual sense as subschemes would sit inside the divisor $Y \subseteq X$. To handle this issue, we introduce Li-Wu's good degenerations of Hilbert schemes [40]. Li-Wu's idea is to blow up the divisor once subschemes sit inside $Y$ (like neck-stretching in Donaldson theory), and we are then reduced to consider subschemes which are 'transversal' to $Y$.

\footnotetext{
${ }^{1}$ Theorem 3.1 ensures we have many such examples.

${ }^{2}$ See Theorem 1.8 and Proposition 1.9 for some partial verification of the existence of relative orientations.

${ }^{3}$ See Definition 6.5 of 8 for the meaning of orientations.
} 
Working with their good degenerations, we study extensions of the above Cases I-III to ideal sheaves cases. In particular, we study the obstruction theory of moduli spaces of relative ideal sheaves (Lemma 5.3) and discuss a gluing formula (Theorem 5.12) based on certain conjectures. We also compute examples on relative $D T_{4}$ virtual cycles (Example 4.1-Example 4.5) based on Definition 5.4, which include

Example 1.3 (Generic quintic in $\mathbb{P}^{4}$, Example 4.4). We take $X=\mathbb{P}^{4}$ which contains a generic quintic 3-fold $Y=Q$ as its anti-canonical divisor, and consider the primitive curve class $[H] \in H_{2}(X, \mathbb{Z})$. Ideal sheaves of curves representing this class have Chern character $c=\left(1,0,0,-P D([H]), \frac{3}{2}\right)$ and we denote their moduli space by $I_{\frac{3}{2}}(X,[H])(\cong G r(2,5))$. The generic quintic $Q$ contains 2875 rigid degree 1 rational curves, and $I_{\frac{3}{2}}(X,[H])$ contains a finite subset $S$ with 2875 points. $I_{\frac{3}{2}}(X,[H]) \backslash S$ has a well-defined restriction morphism to $H i l b^{5}(Q)$. To extend the morphism across those 2875 points, we introduce Li-Wu's expanded pair $X[1]_{0}=X \cup \Delta_{1}, Y[1]_{0}(\cong Q) \subseteq \Delta_{1}$, where $\Delta_{1} \cong \mathbb{P}\left(\left.\mathcal{O}_{Q} \oplus \mathcal{O}_{\mathbb{P}^{4}}(5)\right|_{Q}\right)$, and consider the moduli space $I_{\frac{3}{2}}\left(X[1]_{0},[H]\right)$ of relative ideal sheaves of curves. Geometrically, it is the blowup of $I_{\frac{3}{2}}(X,[H])$ along those 2875 points, i.e.

$$
I_{\frac{3}{2}}\left(X[1]_{0},[H]\right) \cong B l_{S}(G r(2,5)),
$$

where each exceptional divisor corresponds to a $H i l b^{5}\left(\mathbb{P}^{1}\right)$ for each $\mathbb{P}^{1} \subseteq Q$. We then have an injective restriction morphism

$$
\begin{gathered}
I_{\frac{3}{2}}\left(X[1]_{0},[H]\right) \rightarrow \operatorname{Hilb}^{5}\left(Y[1]_{0}\right), \\
\left.I_{C} \mapsto I_{C}\right|_{Y[1]_{0}}
\end{gathered}
$$

with smooth image. Conditions in Definition 5.4 (or Case III) are satisfied, and the relative $D T_{4}$ virtual cycle is the usual fundamental class of the moduli space $I_{\frac{3}{2}}\left(X[1]_{0},[H]\right) \cong B l_{S}(G r(2,5))$.

Proposition 1.4. For $\left(\mathbb{P}^{4}, Q\right)$, we have a restriction morphism

$$
I_{\frac{3}{2}}\left(\mathbb{P}^{4}[1]_{0},[H]\right) \rightarrow \operatorname{Hilb}^{5}(Q)
$$

from the moduli space of relative ideal sheaves of degree 1 rational curves in $\mathbb{P}^{4}$ to the Hilbert scheme of five points in a generic quintic 3 -fold. The relative $D T_{4}$ virtual cycle of $I_{\frac{3}{2}}\left(\mathbb{P}^{4}[1]_{0},[H]\right)$ is the usual fundamental class of the moduli space $I_{\frac{3}{2}}\left(\mathbb{P}^{4}[1]_{0},[H]\right) \cong B l_{S}(G r(2,5))$.

We adapt Li-Wu's good degenerations to torsion sheaves and verify Conjectures 5.8 and 5.9 in the following case.

Example 1.5 (Relative $D T_{4} / D T_{3}$, Example 4.5). Let $X=Y_{1} \times \mathbb{P}^{1}$ which contains $Y=\left(Y_{1} \times 0\right) \sqcup\left(Y_{1} \times \infty\right)$ as an anti-canonical divisor, where $Y_{1}$ is a compact Calabi-Yau 3-fold. We denote $\mathfrak{M}_{c}\left(Y_{1}\right)$ to be a Gieseker moduli space of torsionfree semi-stable sheaves on $Y_{1}$ with Chern character $c \in H^{\text {even }}\left(Y_{1}, \mathbb{Q}\right.$ ) (we assume there is no strictly semi-stable sheaf), and denote $\mathfrak{M}_{c}(X)$ to be the moduli space of sheaves on $X$ which are the push-forward of stable sheaves in $\mathfrak{M}_{c}\left(Y_{1} \times t\right)$ for some $t\left(\mathfrak{M}_{c}(X) \cong \mathfrak{M}_{c}\left(Y_{1}\right) \times \mathbb{P}^{1}\right)$.

To have a well-defined restriction map, we introduce $X[1]_{0}=\Delta_{-1} \cup X \cup \Delta_{1}$, where $\Delta_{ \pm 1} \cong Y_{1} \times \mathbb{P}^{1}$, and consider the relative moduli space $\mathfrak{M}_{c}\left(X[1]_{0}\right)$ with

$$
\mathfrak{M}_{c}\left(X[1]_{0}\right) \cong \mathfrak{M}_{c}\left(Y_{1}\right) \times \mathbb{P}^{1} .
$$


Conjectures 5.8, 5.9 hold and the relative $D T_{4}$ virtual cycle satisfies

$$
\left[\mathfrak{M}_{c}^{r e l}\left(X[1]_{0}\right)\right]^{\text {vir }}=D T_{3}\left(\mathfrak{M}_{c}\left(Y_{1}\right)\right) \cdot\left[\mathbb{P}^{1}\right] \in H_{2}\left(\mathfrak{M}_{c}\left(X[1]_{0}\right), \mathbb{Z}\right),
$$

where $D T_{3}\left(\mathfrak{M}_{c}\left(Y_{1}\right)\right)$ is the Donaldson-Thomas invariant defined by Thomas [54].

Theorem 1.6. For $\left(X=Y_{1} \times \mathbb{P}^{1}, Y=Y_{1} \times\{0, \infty\}\right)$, where $Y_{1}$ is a compact Calabi-Yau 3-fold, we have a restriction map

$$
\mathfrak{M}_{c}\left(X[1]_{0}\right) \cong \mathfrak{M}_{c}\left(Y_{1}\right) \times \mathbb{P}^{1} \rightarrow p t,
$$

from the moduli space of relative torsion sheaves coming from the push-forward of stable sheaves in $\mathfrak{M}_{c}\left(Y_{1} \times t\right)$, where $\mathfrak{M}_{c}\left(Y_{1}\right)$ is a Gieseker moduli space of torsionfree semi-stable sheaves on $Y_{1}$ consisting of no strictly semi-stable sheaf.

The relative $D T_{4}$ virtual cycle exists and satisfies

$$
\left[\mathfrak{M}_{c}^{r e l}\left(X[1]_{0}\right)\right]^{v i r}=D T_{3}\left(\mathfrak{M}_{c}\left(Y_{1}\right)\right) \cdot\left[\mathbb{P}^{1}\right] \in H_{2}\left(\mathfrak{M}_{c}\left(X[1]_{0}\right), \mathbb{Z}\right),
$$

where $D T_{3}\left(\mathfrak{M}_{c}\left(Y_{1}\right)\right)$ is the $D T_{3}$ invariant of $Y_{1}\left(C Y_{3}\right)$ with respect to Chern character $c$.

Finally, we give a coherent description of the orientability issues involved in Cases I-III (Proposition 6.2) and summarize them into the following definition.

Definition 1.7 (Definition6.1). Let $X$ be a smooth projective 4-fold with a smooth anti-canonical divisor $Y \in\left|K_{X}^{-1}\right|$, and let $r: \mathcal{M}_{X} \rightarrow \mathcal{M}_{Y}$ be a well-defined restriction morphism between coarse moduli spaces of simple sheaves on $X$ and $Y$ with fixed Chern classes respectively. In this case, there exists a canonical isomorphism

$$
\alpha:\left(\mathcal{L}_{\mathcal{M}_{X}}\right)^{\otimes 2} \cong r^{*} \mathcal{L}_{\mathcal{M}_{Y}} .
$$

A relative orientation for morphism $r$ consists of a square $\operatorname{root}\left(\left.\mathcal{L}_{\mathcal{M}_{Y}}\right|_{\mathcal{M}_{Y}^{\text {red }}}\right)^{\frac{1}{2}}$ of the determinant line bundle $\left.\mathcal{L}_{\mathcal{M}_{Y}}\right|_{\mathcal{M}_{Y}^{\text {red }}}$ and an isomorphism

$$
\theta:\left.\mathcal{L}_{\mathcal{M}_{X}}\right|_{\mathcal{M}_{X}^{\text {red }}} \cong r^{*}\left(\left.\mathcal{L}_{\mathcal{M}_{Y}}\right|_{\mathcal{M}_{Y}^{\text {red }}}\right)^{\frac{1}{2}}
$$

such that $\theta \otimes \theta \cong \alpha$ holds over $\mathcal{M}_{X}^{\text {red }}$ for the isomorphism $\alpha$.

We then give the following partial verification of the existence of orientations.

Theorem 1.8 (Weak relative orientability, Theorem 6.3). Let $Y$ be a smooth anticanonical divisor in a projective 4-fold $X$ with $\operatorname{Tor}\left(H_{*}(X, \mathbb{Z})\right)=0$, and $E \rightarrow X$ be a complex vector bundle with structure group $S U(N)$, where $N \gg 0$. Let $\mathcal{M}_{X}$ be a coarse moduli scheme of simple holomorphic structures on E, which has a well-defined restriction morphism

$$
r: \mathcal{M}_{X} \rightarrow \mathcal{M}_{Y}
$$

to a proper coarse moduli scheme of simple bundles on $Y$ with fixed Chern classes.

Then there exists a square root $\left(\left.\mathcal{L}_{\mathcal{M}_{Y}}\right|_{\mathcal{M}_{Y}^{\text {red }}}\right)^{\frac{1}{2}}$ of $\left.\mathcal{L}_{\mathcal{M}_{Y}}\right|_{\mathcal{M}_{Y}^{\text {red }}}$ such that

$$
c_{1}\left(\left.\mathcal{L}_{\mathcal{M}_{X}}\right|_{\mathcal{M}_{X}^{\text {red }}}\right)=r^{*} c_{1}\left(\left(\left.\mathcal{L}_{\mathcal{M}_{Y}}\right|_{\mathcal{M}_{Y}^{\text {red }}}\right)^{\frac{1}{2}}\right)
$$

where $\mathcal{L}_{\mathcal{M}_{X}}$ (resp. $\mathcal{L}_{\mathcal{M}_{Y}}$ ) is the determinant line bundle of $\mathcal{M}_{X}$ (resp. $\mathcal{M}_{Y}$ ).

Another partial verification is given as follows.

Proposition 1.9 (Proposition 6.4). We assume $H^{1}\left(\mathcal{M}_{X}, \mathbb{Z}_{2}\right)=0$. Then relative orientations for restriction morphism $r: \mathcal{M}_{X} \rightarrow \mathcal{M}_{Y}$ exist. 


\section{REVIEW OF BASIC FACTS IN DT THEORY}

2.1. Some basic facts in $D T_{4}$ theory. We start with a complex projective CalabiYau 4-fold $\left(X, \mathcal{O}_{X}(1)\right)(\operatorname{Hol}(X)=S U(4))$ with a Ricci-flat Kähler metric $g$ [59], a Kähler form $\omega$, a holomorphic four-form $\Omega$, and a topological bundle with a Hermitian metric $(E, h)$. We define

$$
\left.*_{4}=(\Omega\lrcorner\right) \circ *: \Omega^{0,2}(X, E n d E) \rightarrow \Omega^{0,2}(X, E n d E),
$$

with $*_{4}^{2}=1$, and it splits the corresponding harmonic subspace into (anti-)self-dual parts.

The $D T_{4}$ equations are defined to be

$$
\left\{\begin{array}{l}
F_{+}^{0,2}=0 \\
F \wedge \omega^{3}=0
\end{array}\right.
$$

where the first equation is $F^{0,2}+*_{4} F^{0,2}=0$, and we assume $c_{1}(E)=0$ for simplicity in the moment map equation $F \wedge \omega^{3}=0$.

We denote by $\mathcal{M}^{D T_{4}}(X, g,[\omega], c, h)$ or simply $\mathcal{M}_{c}^{D T_{4}}$ the space of gauge equivalence classes of solutions to the $D T_{4}$ equations on $E$ with $\operatorname{ch}(E)=c$.

We take $\mathcal{M}_{c}^{b d l}$ to be the moduli space of slope-stable holomorphic bundles with fixed Chern character $c$. By the Donaldson-Uhlenbeck-Yau theorem [56], 17], we can identify it with the moduli space of gauge equivalence classes of solutions to the holomorphic HYM equations

$$
\left\{\begin{array}{l}
F^{0,2}=0 \\
F \wedge \omega^{3}=0
\end{array}\right.
$$

By a characteristic class argument, if $c h_{2}(E) \in H^{2,2}(X, \mathbb{C})$, then $F_{+}^{0,2}=0 \Rightarrow F^{0,2}=$ 0 . In particular, if $\mathcal{M}_{c}^{b d l} \neq \emptyset$, then $\mathcal{M}_{c}^{D T_{4}} \cong \mathcal{M}_{c}^{b d l}$ as sets.

The comparison of analytic structures is given by

Theorem 2.1 (Theorem $1.1[13]$ ). We assume $\mathcal{M}_{c}^{b d l} \neq \emptyset$ and fix $d_{A} \in \mathcal{M}_{c}^{D T_{4}}$. Then:

(1) There exists a Kuranishi map $\tilde{\tilde{\kappa}}$ of $\mathcal{M}_{c}^{b d l}$ at $\bar{\partial}_{A}\left(\right.$ the $(0,1)$ part of $\left.d_{A}\right)$ such that $\tilde{\tilde{\kappa}}_{+}$is a Kuranishi map of $\mathcal{M}_{c}^{D T_{4}}$ at $d_{A}$, where

$$
\tilde{\tilde{\kappa}}_{+}=\pi_{+}(\tilde{\tilde{\kappa}}): H^{0,1}(X, E n d E) \stackrel{\tilde{\tilde{\kappa}}}{\longrightarrow} H^{0,2}(X, E n d E) \stackrel{\pi_{+}}{\longrightarrow} H_{+}^{0,2}(X, E n d E)
$$

and $\pi_{+}$is a projection to the self-dual forms.

(2) The closed imbedding between analytic spaces possibly with non-reduced structures $\mathcal{M}_{c}^{\text {bdl }} \hookrightarrow \mathcal{M}_{c}^{D T_{4}}$ is also a homeomorphism between topological spaces.

Remark 2.2. By Proposition 10.10 [13, the map $\tilde{\tilde{\kappa}}$ satisfies $Q_{\text {Serre }}(\tilde{\tilde{\kappa}}, \tilde{\tilde{\kappa}}) \geq 0$, where $Q_{\text {Serre }}$ is the Serre duality pairing on $H^{0,2}(X, E n d E)$.

To define Donaldson type invariants using $\mathcal{M}_{c}^{D T_{4}}$, we need to give them a good compactification (such that it carries a deformation invariant fundamental class). For this purpose, we introduce $\mathcal{M}_{c}\left(X, \mathcal{O}_{X}(1)\right)$ or simply $\mathcal{M}_{c}$ to be the Gieseker moduli space of $\mathcal{O}_{X}(1)$-stable sheaves on $X$ with given Chern character $c$. Motivated by Theorem 2.1 we make the following definition.

Definition 2.3 ([13]). We call a $C^{\infty}$-scheme, $\overline{\mathcal{M}}_{c}^{D T_{4}}$, a generalized $D T_{4}$ moduli space if there exists a homeomorphism

$$
\mathcal{M}_{c} \rightarrow \overline{\mathcal{M}}_{c}^{D T_{4}}
$$


such that at each closed point of $\mathcal{M}_{c}$, say $\mathcal{F}, \overline{\mathcal{M}}_{c}^{D T_{4}}$ is locally isomorphic to $\kappa_{+}^{-1}(0)$, where

$$
\kappa_{+}=\pi_{+} \circ \kappa: \operatorname{Ext}^{1}(\mathcal{F}, \mathcal{F}) \rightarrow \operatorname{Ext}_{+}^{2}(\mathcal{F}, \mathcal{F}),
$$

$\kappa$ is a Kuranishi map at $\mathcal{F}$ and $\operatorname{Ext}_{+}^{2}(\mathcal{F}, \mathcal{F})$ is a half dimensional real subspace of $\operatorname{Ext}^{2}(\mathcal{F}, \mathcal{F})$ on which the Serre duality quadratic form is real and positive definite.

Remark 2.4. The existence of generalized $D T_{4}$ moduli spaces was proved by BorisovJoyce [7]. The authors proved their existence as real analytic spaces in certain cases and defined the corresponding virtual fundamental classes [12, 13]. For fixed data $\left(X, \mathcal{O}_{X}(1), c\right)$, generalized $D T_{4}$ moduli spaces might not be unique.

The proof of Borisov-Joyce's gluing result is divided into two parts. Firstly, they used good local models of $\mathcal{M}_{c}$, i.e. local Darboux charts in the sense of Brav, Bussi and Joyce [9]. Then they chose the half dimensional real subspace $\operatorname{Ext}_{+}^{2}(\mathcal{F}, \mathcal{F})$ appropriately and used partition of unity and homotopical algebra to glue $\kappa_{+}$. The analytic version of BBJ's theorem is as follows.

Theorem 2.5 (Brav-Bussi-Joyce [9], Corollary 5.20; see also Theorem 10.7 [13]). Let $\mathcal{M}_{c}$ be a Gieseker moduli space of stable sheaves on a compact Calabi-Yau 4fold $X$. Then for any closed point $\mathcal{F} \in \mathcal{M}_{c}$, there exist an analytic neighbourhood $U_{\mathcal{F}} \subseteq \mathcal{M}_{c}$, a holomorphic map near the origin

$$
\kappa: \operatorname{Ext}^{1}(\mathcal{F}, \mathcal{F}) \rightarrow \operatorname{Ext}^{2}(\mathcal{F}, \mathcal{F})
$$

such that $Q_{\text {Serre }}(\kappa, \kappa)=0$ and $\kappa^{-1}(0) \cong U_{\mathcal{F}}$ as complex analytic spaces possibly with non-reduced structures, where $Q_{\text {Serre }}$ is the Serre duality pairing on $\operatorname{Ext}^{2}(\mathcal{F}, \mathcal{F})$.

To make sense of virtual fundamental classes of generalized $D T_{4}$ moduli spaces as homology classes in $\mathcal{M}_{c}$ 's, one would in general rely on Joyce's D-manifolds theory [30] or Fukaya-Oh-Ohta-Ono's theory of Kuranishi spaces 22] or Hofer's polyfolds theory [25] (see [58] for a comparison between them). Assuming this part, which is claimed by Borisov-Joyce, we have

Theorem 2.6 (Borisov-Joyce 7], 31]). Let X be a complex projective Calabi-Yau 4-fold, and $\mathcal{M}_{c}$ be a Gieseker moduli space of stable sheaves which is compact (it is true if the degree and rank of sheaves are coprime). Then there exists a generalized $D T_{4}$ moduli space $\overline{\mathcal{M}}_{c}^{D T_{4}}$. If we further assume $\overline{\mathcal{M}}_{c}^{D T_{4}}$ is orientable in the sense of a D-manifold, then the virtual fundamental class of $\overline{\mathcal{M}}_{c}^{D T_{4}}$ exists and is a well-defined homology class 4 i.e.

$$
\left[\overline{\mathcal{M}}_{c}^{D T_{4}}\right]^{\text {vir }} \in H_{*}\left(\mathcal{M}_{c}, \mathbb{Z}\right),
$$

which coincides with earlier definitions of $D T_{4}$ virtual cycles (Definition 5.3, 5.12, 5.14 of 13 ).

We can furthermore define the $\mathrm{DT}_{4}$ invariant by pairing the above cycle with a $\mu$-map as in Definition 5.15 of [13]. With appropriate choice of orientations, $D T_{4}$ invariants are invariant under deformations of complex structures of $X$.

Because of the Serre duality for $\operatorname{Ext}(\mathcal{F}, \mathcal{F})$, the existence of an orientation on a generalized $D T_{4}$ moduli space $\overline{\mathcal{M}}_{c}^{D T_{4}}$ (in the sense of a D-manifold) is equivalent to the existence of a reduction of the structure group of $\left(\mathcal{L}_{\mathcal{M}_{c}}, Q_{\text {Serre }}\right)$ to $S O(1, \mathbb{C})$,

\footnotetext{
${ }^{4}$ It does not depend on the choice of $\overline{\mathcal{M}}_{c}^{D T_{4}}$.
} 
where $\mathcal{L}_{\mathcal{M}_{c}}$ is the determinant line bundle with $\left.\mathcal{L}_{\mathcal{M}_{c}}\right|_{\mathcal{F}} \cong\left(\bigwedge^{\text {top }} \operatorname{Ext}^{\text {even }}(\mathcal{F}, \mathcal{F})\right)^{-1} \otimes$ $\bigwedge^{\text {top }} \operatorname{Ext}^{\text {odd }}(\mathcal{F}, \mathcal{F})$ and $Q_{\text {Serre }}$ is the Serre duality quadratic form on it.

Theorem 2.7 (Theorem 2.2, 14). Let $X$ be a compact Calabi-Yau 4-fold with $H_{\text {odd }}(X, \mathbb{Z})=0$. For any Gieseker moduli space $\mathcal{M}_{c}$ of stable sheaves, the structure group of $\left(\mathcal{L}_{\mathcal{M}_{c}}, Q_{\text {Serre }}\right)$ can be reduced to $S O(1, \mathbb{C})$.

In the case when the Gieseker moduli space $\mathcal{M}_{c}$ on $X$ is smooth (i.e. Kuranishi maps are zero), the obstruction sheaf $O b$ such that $\left.O b\right|_{\mathcal{F}} \cong \operatorname{Ext}^{2}(\mathcal{F}, \mathcal{F})$ is a bundle with Serre duality quadratic form. There exists a real subbundle $O b_{+}$with positive definite quadratic form such that $O b \cong O b_{+} \otimes_{\mathbb{R}} \mathbb{C}$ as vector bundles with quadratic forms. We call $O b_{+}$the self-dual obstruction bundle. By Definition 5.12 of 13 , the virtual fundamental class of $\overline{\mathcal{M}}_{c}^{D T_{4}}$ is the Poincaré dual of the Euler class of the self-dual obstruction bundle (if it is orientable), i.e.

$$
\left[\overline{\mathcal{M}}_{c}^{D T_{4}}\right]^{\text {vir }}=P D\left(e\left(O b_{+}\right)\right) \in H_{*}\left(\mathcal{M}_{c}, \mathbb{Z}\right) .
$$

This motivates later definitions of relative $D T_{4}$ invariants.

2.2. Some basic facts in $D T_{3}$ theory. Moduli spaces of simple sheaves on $C Y_{3}$ 's are locally critical points of holomorphic functions [9], 32], and we can consider the perverse sheaves of vanishing cycles of these functions. The expected cohomology which categorifies $D T_{3}$ invariants is defined by first gluing these local perverse sheaves and then taking its hypercohomology.

Theorem 2.8 (Brav-Bussi-Dupont-Joyce-Szendroi [8], Kiem-Li [33]). Let $Y$ be a Calabi-Yau 3-fold over $\mathbb{C}$, and $\mathcal{M}$ a classical moduli scheme of simple coherent sheaves or simple complexes of coherent sheaves on $Y$, with natural (symmetric) obstruction theory $\phi: \mathcal{E}^{\bullet} \rightarrow \mathbb{L}_{\mathcal{M}}$. Suppose we are given a square root of $\operatorname{det}\left(\mathcal{E}^{\bullet}\right)$.

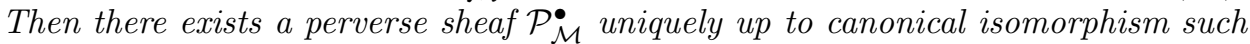
that the Euler characteristic of its hypercohomology is the Donaldson-Thomas invariant [54].

Remark 2.9. When the moduli space $\mathcal{M}$ is smooth (i.e. Kuranishi maps vanish), $\operatorname{det}\left(\mathcal{E}^{\bullet}\right)$ has a natural square root given by the canonical bundle $K_{\mathcal{M}}$. With respect to this square root, the perverse sheaf $\mathcal{P}_{\mathcal{M}}$ is the constant sheaf $\mathbb{C}[\operatorname{dim} \mathcal{M}]$ over $\mathcal{M}$ (see Theorem 6.9 of 8 ).

2.3. An overview of TQFT type structures in $D T_{4}-D T_{3}$ theories. In this subsection, we give an overview of TQFT structures in gauge theories on Calabi-Yau 3 -folds and 4-folds.

We take a smooth (Calabi-Yau) 3-fold $Y$ in a complex projective 4 -fold $X^{+}$as its anti-canonical divisor, and consider a moduli space $\mathfrak{M}_{X^{+}}$of stable bundles with fixed Chern classes on $X^{+}$which has a well-defined restriction morphism

$$
r: \mathfrak{M}_{X^{+}} \rightarrow \mathfrak{M}_{Y}
$$

to a moduli space of stable sheaves on $Y$. This would determine a class $v\left(\mathfrak{M}_{X^{+}}\right) \in$ $\mathbb{H}^{*}\left(\mathcal{P}_{\mathfrak{M}_{Y}}^{\bullet}\right)$.

Given another complex projective 4 -fold $X^{-}$which contains $Y$ as its anticanonical divisor, we form a singular space $X_{0}=X^{+} \cup_{Y} X^{-}$. When $X_{0}$ admits a smooth deformation $X_{t}, X_{t}$ will be a family of $C Y_{4}$ 's provided that the normal bundle of $Y$ in $X^{ \pm}$is trivial. As the perverse sheaf in Theorem 2.8 is self-dual under 
the Verdier duality, i.e. $\mathbb{D}_{\mathfrak{M}_{Y}}\left(\mathcal{P}_{\mathfrak{M}_{Y}}^{\bullet}\right) \cong \mathcal{P}_{\mathfrak{M}_{Y}}^{\bullet}$ [], we can define $\left\langle v\left(\mathfrak{M}_{X^{+}}\right), v\left(\mathfrak{M}_{X^{-}}\right)\right\rangle$ using the Verdier duality on $\mathbb{H}^{*}\left(\mathfrak{M}_{Y}, \mathcal{P}_{\mathfrak{M}_{Y}}\right)$ as long as $\mathfrak{M}_{Y}$ is compact.

Ignoring the stability issue and contributions from general coherent sheaves, we ask the following question, which can be regarded as a complexification of ChernSimons-Donaldson-Floer TQFT structure for 3- and 4-manifolds (see Atiyah [1]).

Question 2.10. What is the relation between $D T_{4}$ invariants and relative $D T_{4}$ invariants, namely, comparing $D T_{4}\left(\mathfrak{M}_{X_{t}}\right)$ with $\left\langle v\left(\mathfrak{M}_{X^{+}}\right), v\left(\mathfrak{M}_{X^{-}}\right)\right\rangle$?

\section{Relative $D T_{4}$ INVARIANTS FOR HOLOMORPHIC BUNDLES}

3.1. Definitions of relative $D T_{4}$ invariants. In this section, we restrict to some good cases and define rigorously the relative $D T_{4}$ invariant mentioned before, i.e. $v\left(\mathfrak{M}_{X^{+}}\right) \in \mathbb{H}^{*}\left(\mathcal{P}_{\mathfrak{M}_{Y}}^{\bullet_{Y}}\right)$. To have a well-defined restriction map, in this section, we assume all Gieseker moduli spaces of semi-stable sheaves on 4-folds consist of slopestable bundles only. This will serve as a model for the later study of relative $D T_{4}$ invariants for ideal sheaves.

Let $Y$ be a smooth anti-canonical divisor of a smooth projective 4 -fold $X, \mathfrak{M}_{X}$ be a Gieseker moduli space which is admissible with respect to $(X, Y)$ (see Definition 1.1), and

$$
r: \mathfrak{M}_{X} \rightarrow \mathfrak{M}_{Y}
$$

be the restriction morphism to a Gieseker moduli space on $Y$. We recall the following criterion which ensures that we have such morphism $r$ in many cases.

Theorem 3.1 (Flenner 21). Let $\left(X, \mathcal{O}_{X}(1)\right)$ be a complex n-dimensional normal projective variety with $\mathcal{O}_{X}(1)$ very ample. We take $\mathcal{F}$ to be an $\mathcal{O}_{X}(1)$-slope semistable torsion-free sheaf of rank $r$. $d$ and $1 \leq c \leq n-1$ are integers such that

$$
\left[\left(\begin{array}{c}
n+d \\
d
\end{array}\right)-c d-1\right] / d>\operatorname{deg}\left(\mathcal{O}_{X}(1)\right) \cdot \max \left(\frac{r^{2}-1}{4}, 1\right) .
$$

Then for a generic complete intersection $Y=H_{1} \cap \cdots \cap H_{c}$ with $H_{i} \in\left|\mathcal{O}_{X}(d)\right|$, $\left.\mathcal{F}\right|_{Y} \triangleq \mathcal{F} \otimes_{\mathcal{O}_{X}} \mathcal{O}_{Y}$ is $\left.\mathcal{O}_{X}(1)\right|_{Y}$-slope semi-stable on $Y$.

Remark 3.2. For $X=\mathbb{P}^{4}, \mathcal{O}_{X}(1)=\mathcal{O}_{\mathbb{P}^{4}}(1)$ is very ample. We take $c=1$ and $d=5$. Then any rank $r \leq 9$ semi-stable sheaf on $\mathbb{P}^{4}$ remains semi-stable when restricted to a generic quintic 3 -fold inside.

The deformation-obstruction theory associated to the restriction morphism $r$ is described by the following exact sequence.

Lemma 3.3. We take a stable bundle $E \in \mathfrak{M}_{X}$ and assume $Y$ is connected. Then we have a long exact sequence,

$$
\begin{aligned}
& 0 \rightarrow H^{1}\left(X, E n d E \otimes K_{X}\right) \rightarrow H^{1}(X, E n d E) \rightarrow H^{1}\left(Y,\left.E n d E\right|_{Y}\right) \\
& \rightarrow H^{2}\left(X, E n d E \otimes K_{X}\right) \rightarrow H^{2}(X, E n d E) \rightarrow H^{2}\left(Y,\left.E n d E\right|_{Y}\right) \\
& \rightarrow H^{3}\left(X, E n d E \otimes K_{X}\right) \rightarrow H^{3}(X, E n d E) \rightarrow 0 .
\end{aligned}
$$

Proof. We tensor $0 \rightarrow \mathcal{O}_{X}(-Y) \rightarrow \mathcal{O}_{X} \rightarrow \mathcal{O}_{Y} \rightarrow 0$ with EndE and take its cohomology.

We note that the transpose of the above sequence with respect to Serre duality pairing on $X$ and $Y$ remains the same (see also [19]). This will be the key property used in the following definitions of relative $D T_{4}$ invariants. 
Case I (when $\mathfrak{M}_{Y}$ is of expected dim). If we assume $H^{1}\left(Y,\left.E n d E\right|_{Y}\right)=0$ for any $E \in \mathfrak{M}_{X}$, then $H^{2}\left(Y,\left.E n d E\right|_{Y}\right)=0$ and $\mathfrak{M}_{Y}$ contains components of finite points, labeled by $E_{1}, \ldots, E_{m}$ which come from restrictions of bundles on $X$. We denote $\mathfrak{M}_{X, E_{i}}$ to be components of $\mathfrak{M}_{X}$ such that $r\left(\mathfrak{M}_{X, E_{i}}\right)=E_{i}$. By Lemma 3.3. we have canonical isomorphisms

$$
H^{3}(X, E n d E)^{*} \cong H^{1}(X, E n d E), \quad H^{2}(X, E n d E)^{*} \cong H^{2}(X, E n d E) .
$$

In fact, one can furthermore show that $\mathfrak{M}_{X, E_{i}}$ has a (-2)-shifted symplectic structure in the sense of PTVV [52] (if $X$ is also Fano). Since by section 3.2 of Calaque [11] (see also Example 3.4 of [11]), the restriction morphism $\operatorname{Bun}_{G}(X) \rightarrow \operatorname{Bun}_{G}(Y)$ for (derived) moduli stacks of bundles defines a Lagrangian in a $(-1)$-shifted symplectic derived stack. We restrict to the open locus of stable bundles as in [7] (Borisov-Joyce restricted to locus of Gieseker stable sheaves) and then recover the classical restriction map $r: \mathfrak{M}_{X} \rightarrow \mathfrak{M}_{Y}$ (with image in $\left\{E_{1}, \ldots, E_{m}\right\}$ ). Now by our assumption, $\left\{E_{1}, \ldots, E_{m}\right\}$ are finite of points in the derived sense as all $E x t^{*}$ (trace-free part) vanish. By Example 2.3 of [11, the Lagrangian structure on $r: \mathfrak{M}_{X} \rightarrow\left\{E_{1}, \ldots, E_{m}\right\}$ is nothing but a $(-2)$-shifted symplectic structure on $\mathfrak{M}_{X}$ (each connected component $\mathfrak{M}_{X, E_{i}}$ also has such structure). Analogous to Theorem 2.6. we can define the relative $D T_{4}$ virtual cycle $\left[\mathfrak{M}_{X, E_{i}}^{r e l}\right]^{\text {vir }} \in H_{n}\left(\mathfrak{M}_{X, E_{i}}, \mathbb{Z}_{2}\right)$ with $n=1-\chi(X, E n d E)$. The cycle will be defined over integers if the associated D-manifold of $\mathfrak{M}_{X, E_{i}}$ is orientable.

Since the virtual dimension is not zero in general, we introduce a $\mu$-map as in Definition 5.15 of $[13$.

Definition 3.4. We denote the universal sheaf of $\mathfrak{M}_{X}$ by $\mathfrak{F} \rightarrow \mathfrak{M}_{X} \times X$. The $\mu$-map is

$$
\begin{gathered}
\mu: H_{*}(X) \otimes \mathbb{Z}\left[x_{1}, x_{2}, \ldots\right] \rightarrow H^{*}\left(\mathfrak{M}_{X}\right), \\
\mu(\gamma, P)=P\left(c_{1}(\mathfrak{F}), c_{2}(\mathfrak{F}), \ldots\right) / \gamma
\end{gathered}
$$

Pairing virtual cycles with $\mu$-maps defines the relative invariants.

Definition 3.5. Let $\mathfrak{M}_{X}$ be a Gieseker moduli space of semi-stable sheaves which is admissible with respect to $(X, Y)$ (see Definition 1.1), and $r: \mathfrak{M}_{X} \rightarrow \mathfrak{M}_{Y}$ be the restriction morphism.

We assume $H^{1}\left(Y,\left.E n d E\right|_{Y}\right)=0$ for any $E \in \mathfrak{M}_{X}$ and $X$ is Fano. Then the relative $D T_{4}$ invariant is a map

$$
v\left(\mathfrak{M}_{X}\right): \operatorname{Sym}^{*}\left(H_{*}(X, \mathbb{Z}) \otimes \mathbb{Z}\left[x_{1}, x_{2}, \ldots\right]\right) \rightarrow \mathbb{H}^{*}\left(\mathfrak{M}_{Y}, \mathcal{P}_{\mathfrak{M}_{Y}}^{\bullet}\right)
$$

such that

$$
v\left(\mathfrak{M}_{X}\right)\left(\left(\gamma_{1}, P_{1}\right),\left(\gamma_{2}, P_{2}\right), \ldots\right)=\sum_{i=1}^{m}\left\langle\left[\mathfrak{M}_{X, E_{i}}^{r e l}\right]^{v i r}, \mu\left(\gamma_{1}, P_{1}\right) \cup \mu\left(\gamma_{2}, P_{2}\right) \cup \ldots\right\rangle E_{i},
$$

where $\langle$,$\rangle denotes the natural pairing between homology and cohomology classes$ and $\left\{E_{i}\right\}_{1 \leq i \leq m}$ are taken as a basis of $H^{*}\left(r\left(\mathfrak{M}_{X}\right)\right)$.

Case II (when $\mathfrak{M}_{X}$ and $\mathfrak{M}_{Y}$ are smooth and $r$ is surjective). We assume $\mathfrak{M}_{X}$ and $\mathfrak{M}_{Y}$ are smooth (i.e. all Kuranishi maps are zero) and the restriction map $r$ is surjective. By Lemma 3.3, we get a canonical isomorphism

$$
H^{2}(X, E n d E)^{*} \cong H^{2}(X, E n d E)
$$


which endows $H^{2}(X, E n d E)$ with a non-degenerate quadratic form, and a short exact sequence

$$
0 \rightarrow H^{3}(X, E n d E)^{*} \rightarrow H^{1}(X, E n d E) \rightarrow H^{1}\left(Y,\left.E n d E\right|_{Y}\right) \rightarrow 0 .
$$

Counting dimensions, we have

$$
2 h^{1}(X, E n d E)-h^{2}(X, E n d E)=h^{1}\left(Y,\left.E n d E\right|_{Y}\right)-\chi(X, E n d E)+1,
$$

which is a constant on components of $\mathfrak{M}_{X}$ by assumption. Similar to (2), the selfdual subbundle of the obstruction bundle $O b_{\mathfrak{M}_{X}}$ exists. If it is also orientable, we define the relative $D T_{4}$ virtual cycle $\left[\mathfrak{M}_{X}^{\text {rel }}\right]^{\text {vir }} \in H_{n}\left(\mathfrak{M}_{X}, \mathbb{Z}\right)$ to be the Euler class of the self-dual obstruction bundle, where $n=h^{1}\left(Y,\left.E n d E\right|_{Y}\right)-\chi(X, E n d E)+1$.

Definition 3.6. Let $\mathfrak{M}_{X}$ be a Gieseker moduli space of semi-stable sheaves which is admissible with respect to $(X, Y)$ (see Definition 1.1), and $r: \mathfrak{M}_{X} \rightarrow \mathfrak{M}_{Y}$ be the restriction morphism.

We assume $r$ is surjective between smooth moduli spaces. Then the relative $D T_{4}$ invariant is a map

$$
v\left(\mathfrak{M}_{X}\right): \operatorname{Sym}^{*}\left(H_{*}(X, \mathbb{Z}) \otimes \mathbb{Z}\left[x_{1}, x_{2}, \ldots\right]\right) \rightarrow H^{*}\left(\mathfrak{M}_{Y}\right)
$$

such that

$$
v\left(\mathfrak{M}_{X}\right)\left(\left(\gamma_{1}, P_{1}\right),\left(\gamma_{2}, P_{2}\right), \ldots\right)(\alpha)=\left\langle\left[\mathfrak{M}_{X}^{r e l}\right]^{v i r},\left(r^{*} \alpha\right) \cup \mu\left(\gamma_{1}, P_{1}\right) \cup \mu\left(\gamma_{2}, P_{2}\right) \cup \ldots\right\rangle,
$$

where $\alpha \in H^{*}\left(\mathfrak{M}_{Y}\right)$ and we identify $H^{*}\left(\mathfrak{M}_{Y}\right) \cong H^{*}\left(\mathfrak{M}_{Y}\right)^{*}$ via Poincaré pairing; $\langle$,$\rangle denotes the natural pairing between homology and cohomology classes.$

Case III (when $\mathfrak{M}_{X}$ and $\mathfrak{M}_{Y}$ are smooth and $r$ is injective). We assume $\mathfrak{M}_{X}$ and $\mathfrak{M}_{Y}$ are smooth (i.e. all Kuranishi maps are zero) and the restriction map $r$ is injective. By Lemma 3.3. we get $H^{3}(X, E n d E)=0$ and an exact sequence

$$
\begin{aligned}
& 0 \rightarrow H^{1}(X, E n d E) \rightarrow H^{1}\left(Y,\left.E n d E\right|_{Y}\right) \rightarrow H^{2}(X, E n d E)^{*} \\
\rightarrow & H^{2}(X, E n d E) \rightarrow H^{1}\left(Y,\left.E n d E\right|_{Y}\right)^{*} \rightarrow H^{1}(X, E n d E)^{*} \rightarrow 0 .
\end{aligned}
$$

This determines a surjective map

$$
s: O b_{\mathfrak{M}_{X}} \rightarrow \mathcal{N}_{\mathfrak{M}_{X} / \mathfrak{M}_{Y}}^{*}
$$

and a non-degenerate quadratic form on the reduced bundle $\operatorname{Ob}_{\mathfrak{M}_{X}}^{\text {red }} \triangleq \operatorname{Ker}(s)$, where $O b_{\mathfrak{M}_{X}}$ is the obstruction bundle of $\mathfrak{M}_{X}$ with $\left.O b_{\mathfrak{M}_{X}}\right|_{E}=H^{2}(X$, EndE) and $\mathcal{N}_{\mathfrak{M}_{X} / \mathfrak{M}_{Y}}^{*}$ is the conormal bundle of $\mathfrak{M}_{X}$ inside $\mathfrak{M}_{Y}$.

Then if the self-dual subbundle of $O b_{\mathfrak{M}_{X}}^{\text {red }}$ is orientable, we define the relative $D T_{4}$ virtual cycle $\left[\mathfrak{M}_{X}^{\text {rel }}\right]^{\text {vir }} \in H_{n}\left(\mathfrak{M}_{X}, \mathbb{Z}\right)$ to be the Euler class of it, where the virtual dimension is $n=2 h^{1}(X, E n d E)-\left(h^{2}(X, E n d E)-\operatorname{codim}_{\mathfrak{M}_{Y}}\left(\mathfrak{M}_{X}\right)\right)=$ $h^{1}\left(Y,\left.E n d E\right|_{Y}\right)-\chi(X, E n d E)+1$. Note that when $\mathfrak{M}_{X}$ is smooth, $r$ is injective and a neighbourhood of $r\left(\mathfrak{M}_{X}\right) \subseteq \mathfrak{M}_{Y}$ is smooth. We could also define $\left[\mathfrak{M}_{X}^{\text {rel }}\right]^{\text {vir }}$ in a similar way.

Definition 3.7. Let $\mathfrak{M}_{X}$ be a Gieseker moduli space of semi-stable sheaves which is admissible with respect to $(X, Y)$ (see Definition 1.1), and $r: \mathfrak{M}_{X} \rightarrow \mathfrak{M}_{Y}$ be the restriction morphism.

We assume $r$ is injective between smooth moduli spaces (at least when restricted to a neighbourhood of $r\left(\mathfrak{M}_{X}\right)$ in $\left.\mathfrak{M}_{Y}\right)$. Then the relative $D T_{4}$ invariant is a map

$$
v\left(\mathfrak{M}_{X}\right): \operatorname{Sym}^{*}\left(H_{*}(X, \mathbb{Z}) \otimes \mathbb{Z}\left[x_{1}, x_{2}, \ldots\right]\right) \rightarrow H^{*}\left(\mathfrak{M}_{Y}\right)
$$


such that

$$
v\left(\mathfrak{M}_{X}\right)\left(\left(\gamma_{1}, P_{1}\right),\left(\gamma_{2}, P_{2}\right), \ldots\right)(\alpha)=\left\langle\left[\mathfrak{M}_{X}^{r e l}\right]^{v i r},\left(r^{*} \alpha\right) \cup \mu\left(\gamma_{1}, P_{1}\right) \cup \mu\left(\gamma_{2}, P_{2}\right) \cup \ldots\right\rangle,
$$

where $\alpha \in H^{*}\left(\mathfrak{M}_{Y}\right)$ and we identify $H^{*}\left(\mathfrak{M}_{Y}\right) \cong H^{*}\left(\mathfrak{M}_{Y}\right)^{*}$ via Poincaré pairing; $\langle$,$\rangle denotes the natural pairing between homology and cohomology classes.$

Remark 3.8. It is easy to check that Definitions 3.5, 3.6 and 3.7 are all compatible.

In general, one could consider moduli spaces of complexes of simple sheaves 42 . on a complex projective 4 -fold $X$ and resolve complexes of sheaves by complexes of holomorphic bundles. Then there will be a natural restriction morphism to a moduli of simple bundles on an anti-canonical divisor of $X$. A similar long exact sequence in Lemma 3.3 still works, and we could study virtual cycle constructions as in Cases I-III.

Endomorphisms of $D T_{3}$ cohomologies from relative $D T_{4}$ invariants. By considering the trace-free version of Lemma 3.3. the above definitions extend to any disconnected divisor $Y \subseteq X$. We are particularly interested in the case when $X=Y_{1} \times \mathbb{P}^{1}$, where $Y_{1}$ is a compact Calabi-Yau 3-fold. Then $Y=\left(Y_{1} \times 0\right) \sqcup\left(Y_{1} \times \infty\right)$ will be a smooth anti-canonical divisor of $X$, and the relative $D T_{4}$ invariant in general is a map

$$
\begin{aligned}
v\left(\mathfrak{M}_{X}\right) & : \operatorname{Sym}^{*}\left(H_{*}(X, \mathbb{Z}) \otimes \mathbb{Z}\left[x_{1}, x_{2}, \ldots\right]\right) \\
\rightarrow & \mathbb{H}^{*}\left(\mathfrak{M}_{Y}, \mathcal{P}_{\mathfrak{M}_{Y}}^{\bullet}\right) \cong \mathbb{H}^{*}\left(\mathfrak{M}_{Y_{1}}, \mathcal{P}_{\mathfrak{M}_{Y_{1}}}^{\bullet}\right) \otimes \mathbb{H}^{*}\left(\mathfrak{M}_{Y_{1}}, \mathcal{P}_{\mathfrak{M}_{Y_{1}}}^{\bullet}\right) .
\end{aligned}
$$

By the Verdier duality and $\mathbb{D}_{\mathfrak{M}_{Y_{1}}}\left(\mathcal{P}_{\mathfrak{M}_{Y_{1}}}\right) \cong \mathcal{P}_{\mathfrak{M}_{Y_{1}}}$, we have

$$
\mathbb{H}^{*}\left(\mathfrak{M}_{Y_{1}}, \mathcal{P}_{\mathfrak{M}_{Y_{1}}}^{\bullet}\right) \cong \mathbb{H}^{*}\left(\mathfrak{M}_{Y_{1}}, \mathcal{P}_{\mathfrak{M}_{Y_{1}}}^{\bullet}\right)^{*}
$$

Thus a relative $D T_{4}$ invariant of $\left(Y_{1} \times \mathbb{P}^{1}, Y_{1} \times\{0, \infty\}\right)$ determines some endomorphisms of the $D T_{3}$ cohomology

$$
v\left(\mathfrak{M}_{X}\right): \operatorname{Sym}^{*}\left(H_{*}\left(Y_{1}\right)[t] /\left(t^{2}\right) \otimes \mathbb{Z}\left[x_{1}, x_{2}, \ldots\right]\right) \rightarrow \operatorname{End}_{\mathbb{C}}\left(\mathbb{H}^{*}\left(\mathfrak{M}_{Y_{1}}, \mathcal{P}_{\mathfrak{M}_{Y_{1}}}\right)\right)
$$

for any Calabi-Yau 3-fold $Y_{1}$. In general, the above endomorphisms should be used to establish the gluing formula mentioned in Question 2.10 (see 35] for the real 4-3 dimensional picture).

3.2. Li-Qin's examples. We have Li-Qin's examples when conditions in Cases II and III are satisfied [41. Let $Y$ be a generic smooth hyperplane section in $X=\mathbb{P}^{1} \times \mathbb{P}^{3}$ of bi-degree $(2,4)$ :

$$
\begin{gathered}
c=[1+(-1,1)] \cdot\left[1+\left(\epsilon_{1}+1, \epsilon_{2}-1\right)\right], \\
\left.c\right|_{Y}=\left[1+\left.(-1,1)\right|_{Y}\right] \cdot\left[1+\left.\left(\epsilon_{1}+1, \epsilon_{2}-1\right)\right|_{Y}\right], \\
k=\left(1+\epsilon_{1}\right)\left(\begin{array}{c}
5-\epsilon_{2} \\
3
\end{array}\right)-1, \quad \epsilon_{1}, \epsilon_{2}=0,1, \quad L_{r}=\mathcal{O}_{\mathbb{P}^{1} \times \mathbb{P}^{3}}(1, r) .
\end{gathered}
$$

We denote $\mathfrak{M}_{c}\left(L_{r}\right)$ to be the moduli space of $L_{r}$-slope stable rank-2 bundles on $X$ with a Chern class $c$ and $\overline{\mathfrak{M}}_{\left.c\right|_{Y}}\left(\left.L_{r}\right|_{Y}\right)$ to be the moduli space of Gieseker $\left.L_{r}\right|_{Y^{-}}$ semi-stable rank-2 torsion-free sheaves on $Y$ with Chern class $\left.c\right|_{Y}$.

Lemma 3.9 (Li-Qin 41). (i) If

$$
\frac{4\left(2-\epsilon_{2}\right)}{2+2 \epsilon_{1}+\epsilon_{2}}<r<\frac{4\left(2-\epsilon_{2}\right)}{\epsilon_{1} \epsilon_{2}}
$$


then $\overline{\mathfrak{M}}_{\left.c\right|_{Y}}\left(\left.L_{r}\right|_{Y}\right) \cong \mathbb{P}^{k}$ and consists of rank-2 stable bundles. Furthermore, the restriction map

$$
r: \mathfrak{M}_{c}\left(L_{r}\right) \rightarrow \overline{\mathfrak{M}}_{\left.c\right|_{Y}}\left(\left.L_{r}\right|_{Y}\right)
$$

is well-defined and isomorphic between projective varieties.

(ii) If $0<r<\frac{4\left(2-\epsilon_{2}\right)}{2+2 \epsilon_{1}+\epsilon_{2}}$, then $\mathfrak{M}_{c}\left(L_{r}\right)$ and $\overline{\mathfrak{M}}_{\left.c\right|_{Y}}\left(\left.L_{r}\right|_{Y}\right)$ are empty.

Proposition 3.10. In the above example, for any stable bundle $E \in \mathfrak{M}_{c}\left(L_{r}\right)$ on $X=\mathbb{P}^{1} \times \mathbb{P}^{3}$,

$$
\begin{gathered}
\operatorname{Ext}_{X}^{1}(E, E) \cong \operatorname{Ext}_{Y}^{1}\left(\left.E\right|_{Y},\left.E\right|_{Y}\right) \cong \operatorname{Ext}_{Y}^{2}\left(\left.E\right|_{Y},\left.E\right|_{Y}\right)^{*} \cong \mathbb{C}^{k}, \\
\operatorname{Ext}_{X}^{i}(E, E)=0, \quad \text { if } i \geq 2 .
\end{gathered}
$$

The relative $D T_{4}$ virtual cycle $\left[\mathfrak{M}_{c}^{r e l}\left(L_{r}\right)\right]^{\text {vir }}=\left[\mathfrak{M}_{c}\left(L_{r}\right)\right] \in H_{2 k}\left(\mathfrak{M}_{c}\left(L_{r}\right), \mathbb{Z}\right)$.

\section{Computational examples of Relative $D T_{4}$ invariants FOR IDEAL SHEAVES}

In the above section, we studied relative $D T_{4}$ invariants for holomorphic bundles. To formulate the gluing formula, we need to have a good understanding of how stable sheaves could be degenerated into a union of stable sheaves on irreducible components of degenerated varieties. At this moment, we will restrict ourselves to the ideal sheaves case where degenerations have simpler behavior.

We take a simple degeneration $\pi: \mathcal{X} \rightarrow C$ of projective manifolds over a pointed smooth curve $(C, 0 \in C)$, i.e. (1) $\mathcal{X}$ is smooth, $\pi$ is projective and smooth away from the central fiber $X_{0}=\pi^{-1}(0)$. (2) $X_{0}$ is a union of two smooth irreducible components $X_{+}, X_{-}$intersecting transversally along a smooth divisor $Y$. When generic fibers $X_{t}$ are Calabi-Yau 4-folds and $Y$ is an anti-canonical divisor of $X_{+}$, $X_{-}$, we will study relative $D T_{4}$ invariants of ideal sheaves for pairs $\left(X_{ \pm}, Y\right)$ and discuss their relations with $D T_{4}$ invariants of $X_{t}, t \neq 0$. The basic technique is the degeneration method developed by J. Li and B. Wu [37, [38, [40, [57.

$\mathrm{Li}-\mathrm{Wu}$ 's construction will be recalled in the appendix, and the associated obstruction theory is studied there. In this section, we concentrate on computational examples of relative $D T_{4}$ virtual cycles for ideal sheaves based on the extension (Definition 5.4) of constructions for bundles (Cases I-III).

Example 4.1 (Ideal sheaves of one point). We take a compact simply connected 4fold $5 X_{+}$which contains a smooth Calabi-Yau 3-fold $Y$ as its anti-canonical divisor. We consider the moduli space $I_{1}\left(X_{+}, 0\right)$ of structure sheaves of one point (it is equivalent to consider ideal sheaves of one point) which has a well-defined restriction map to $Y$ if points sit inside $X_{+} \backslash Y$.

To extend the map to the whole moduli space, we introduce Li-Wu's expanded pair; i.e. we consider $X_{+}[1]_{0}=X_{+} \cup_{Y} \Delta, Y[1]_{0} \subseteq \Delta$, where $\Delta \cong \mathbb{P}\left(\mathcal{O}_{Y} \oplus \mathcal{N}_{Y / X_{+}}\right)$ and form the moduli space $I_{1}\left(X_{+}[1]_{0}, 0\right)\left(\cong X_{+}\right)$of relative structure sheaf of one point, which is the union of $X_{+} \backslash Y$ with the $\mathbb{C}^{*}$-equivalence classes of points in $\Delta \backslash((Y \times 0) \cup(Y \times \infty))$. By the Koszul resolution and Serre duality, we have canonical isomorphism

$$
\operatorname{Ext}^{*}\left(\mathcal{O}_{P}, \mathcal{O}_{P}\right) \cong \bigwedge^{*}\left(\left.T X_{+}\right|_{P}\right)
$$

\footnotetext{
${ }^{5}$ Note that any compact Fano manifold is simply connected.
} 
Then the obstruction bundle $O b=\bigwedge^{2} T X_{+}$has a non-degenerate quadratic form only when it is restricted to $X_{+} \backslash Y$ and $\Delta \backslash((Y \times 0) \cup(Y \times \infty))$, but they do not glue to become a non-degenerate quadratic form on $\mathrm{Ob} \rightarrow X_{+}$as $c_{1}(\mathrm{Ob}) \neq 0$.

If we assume $K_{X_{+}}$has a square $\operatorname{root} K_{X_{+}}^{\frac{1}{2}}$ (see the appendix) and forms $\widetilde{O b} \triangleq$ $\bigwedge^{2} T X_{+} \otimes K_{X_{+}}^{\frac{1}{2}}$, then there exists a non-degenerate quadratic form

$$
\left(\bigwedge^{2} T X_{+} \otimes K_{X_{+}}^{\frac{1}{2}}\right) \otimes\left(\bigwedge^{2} T X_{+} \otimes K_{X_{+}}^{\frac{1}{2}}\right) \rightarrow \mathcal{O}_{X_{+}}
$$

As $\pi_{1}(X)=0$, the self-dual obstruction bundle $\widetilde{O b_{+}}$is orientable, and Conjectures 5.8, 5.9 hold for this case. To calculate the Euler class $e\left(\widetilde{O b}_{+}\right)$, we consider the case when $X_{+}=Y_{1} \times \mathbb{P}^{1}$ and $Y=\left(Y_{1} \times 0\right) \sqcup\left(Y_{1} \times \infty\right)$, where $Y_{1}$ is a smooth Calabi-Yau 3 -fold. Then

$$
\bigwedge^{2} T X_{+} \otimes K_{X_{+}}^{\frac{1}{2}} \cong\left(\bigwedge^{2} T Y_{1} \otimes \mathcal{O}_{\mathbb{P}^{1}}(-1)\right) \oplus\left(T Y_{1} \otimes \mathcal{O}_{\mathbb{P}^{1}}(1)\right)
$$

where both factors are maximal isotropic subbundles of $\widetilde{O b}_{+}$. By [20] or Lemma 5.13 of [13],

$$
e\left(\widetilde{O b_{+}}\right)= \pm\left(c_{3}\left(Y_{1}\right)+c_{2}\left(Y_{1}\right) \cdot c_{1}\left(\mathcal{O}_{\mathbb{P}^{1}}(1)\right)\right)= \pm\left(c_{3}\left(X_{+}\right)-\frac{1}{2} c_{1}\left(X_{+}\right) \cdot c_{2}\left(X_{+}\right)\right),
$$

where the sign depends on the orientation of $\widetilde{O b_{+}}$. The relative $D T_{4}$ virtual cycle for structure sheaves of one point is the Poincaré dual of $e\left(\widetilde{O b}_{+}\right)$(2).

We then consider examples of ideal sheaves of curves.

Example 4.2. Let $Q \subseteq \mathbb{P}^{4}$ be a smooth generic quintic 3 -fold. We take $X_{+}=$ $Q \times \mathbb{P}^{1}$ with an anti-canonical divisor $Y=(Q \times 0) \sqcup(Q \times \infty)$. Then $H_{2}\left(X_{+}, \mathbb{Z}\right) \cong$ $H_{2}(Q, \mathbb{Z}) \oplus H_{2}\left(\mathbb{P}^{1}, \mathbb{Z}\right) \cong \mathbb{Z} \oplus \mathbb{Z}$.

(i) We first fix the curve class to be the generator $[H] \in H_{2}\left(\mathbb{P}^{1}, \mathbb{Z}\right) \subseteq H_{2}\left(X_{+}, \mathbb{Z}\right)$. The moduli space $I_{0}\left(X_{+},[H]\right)(\cong Q)$ of ideal sheaves of curves with Chern character $c=(1,0,0,-P D([H]), 0)$ consists of ideal sheaves of curves of type $\{p t\} \times \mathbb{P}^{1}$, $p t \in Q$. We have a restriction morphism

$$
\begin{gathered}
r: I_{0}\left(X_{+},[H]\right) \rightarrow I_{1}(Q, 0) \times I_{1}(Q, 0) \cong Q \times Q, \\
I_{\{p t\} \times \mathbb{P}^{1}} \mapsto\left(I_{p t}, I_{p t}\right),
\end{gathered}
$$

to the moduli space of ideal sheaves of one point in $Y ; r$ is the diagonal embedding if we identify $I_{0}\left(X_{+},[H]\right) \cong Q$. By direct calculations, for any $I_{C} \in I_{0}\left(X_{+},[H]\right)$, we have

$$
\operatorname{Ext}_{X_{+}}^{i}\left(I_{C}, I_{C}\right) \cong \mathbb{C}^{3}, i=1,2, \quad \operatorname{Ext}_{X_{+}}^{3}\left(I_{C}, I_{C}\right) \cong \mathbb{C}, \quad \operatorname{Ext}_{X_{+}}^{4}\left(I_{C}, I_{C}\right)=0 .
$$

Analogous to Lemma 5.3, we have a long exact sequence

$$
\begin{aligned}
& 0 \rightarrow \operatorname{Ext}_{X_{+}}^{1}\left(I_{C}, I_{C}\right) \rightarrow \operatorname{Ext}_{Y}^{1}\left(\left.I_{C}\right|_{Y},\left.I_{C}\right|_{Y}\right) \rightarrow \operatorname{Ext}_{X_{+}}^{2}\left(I_{C}, I_{C} \otimes K_{X_{+}}\right) \\
\rightarrow & \operatorname{Ext}_{X_{+}}^{2}\left(I_{C}, I_{C}\right) \rightarrow \operatorname{Ext}_{Y}^{2}\left(\left.I_{C}\right|_{Y},\left.I_{C}\right|_{Y}\right) \rightarrow \operatorname{Ext}_{X_{+}}^{3}\left(I_{C}, I_{C} \otimes K_{X_{+}}\right) \rightarrow 0 .
\end{aligned}
$$

This determines a surjective morphism

$$
s: O b \rightarrow \mathcal{N}_{Q / Q \times Q}
$$

from the obstruction bundle $O b$ with $\left.O b\right|_{I_{C}}=\operatorname{Ext}_{X_{+}}^{2}\left(I_{C}, I_{C}\right)$ to the conormal bundle of $I_{0}\left(X_{+},[H]\right)$ in $I_{1}(Q, 0)$. Furthermore, $r k(O b)=\operatorname{codim}(Q, Q \times Q)=3$, and 
conditions in Definition 5.4 are satisfied. The relative $D T_{4}$ virtual cycle is the usual fundamental class of the moduli space, i.e. $\left[I_{0}\left(X_{+},[H]\right)\right] \in H_{6}\left(I_{0}\left(X_{+},[H]\right), \mathbb{Z}\right)$.

(ii) If we fix the curve class to be the generator $\left[H_{Q}\right] \in H_{2}(Q, \mathbb{Z}) \subseteq H_{2}\left(X_{+}, \mathbb{Z}\right)$, the moduli space $I_{1}\left(X_{+},\left[H_{Q}\right]\right)$ of ideal sheaves of curves in $X_{+}=Q \times \mathbb{P}^{1}$ with Chern character $c=\left(1,0,0,-P D\left(\left[H_{Q}\right]\right),-1\right)$ can be identified with the product of $\mathbb{P}^{1}$ with the moduli space of primitive rational curves in $Q$ (which consists of 2875 rigid curves for a generic $Q \subseteq \mathbb{P}^{4}[15]$ ), i.e.

$$
I_{1}\left(X_{+},\left[H_{Q}\right]\right) \cong \bigsqcup_{2875} \mathbb{P}^{1}
$$

Curves in $\bigsqcup_{2875} \mathbb{C}^{*}$ have well-defined restriction to trivial line bundles on $(Q \times 0) \sqcup(Q \times \infty)$. For curves in $\bigsqcup_{2875}\{0, \infty\} \subseteq I_{1}\left(X_{+},\left[H_{Q}\right]\right)$, we introduce Li-Wu's expanded pair to define the restriction map. We denote $X_{+}[1]_{0}=\Delta_{-1} \cup$ $X_{+} \cup \Delta_{1}$, where $\Delta_{ \pm 1} \cong Q \times \mathbb{P}^{1}$, and consider the moduli space $I_{1}\left(X_{+}[1]_{0},\left[H_{Q}\right]\right)$ of relative ideal sheaves of curves (normal to the divisor $(Q \times 0) \sqcup(Q \times \infty))$. $I_{1}\left(X_{+}[1]_{0},\left[H_{Q}\right]\right)$ is the union of $\bigsqcup_{2875} \mathbb{C}^{*}$ with $\mathbb{C}^{*}$-equivalence classes of curves inside $\Delta_{ \pm 1} \backslash((Q \times 0) \sqcup(Q \times \infty))$, i.e.

$$
I_{1}\left(X_{+}[1]_{0},\left[H_{Q}\right]\right) \cong \bigsqcup_{2875} \mathbb{P}^{1}
$$

We then have a restriction map

$$
\begin{aligned}
& I_{1}\left(X_{+}[1]_{0},\left[H_{Q}\right]\right) \rightarrow\left\{\mathcal{O}_{Q \times 0}\right\} \sqcup\left\{\mathcal{O}_{Q \times \infty}\right\}, \\
& \left.I_{C} \mapsto I_{C}\right|_{(Q \times 0) \sqcup(Q \times \infty)}=\left(\mathcal{O}_{Q \times 0}, \mathcal{O}_{Q \times \infty}\right)
\end{aligned}
$$

to the moduli space of trivial line bundles on $(Q \times 0) \sqcup(Q \times \infty) \subseteq \Delta_{-1} \sqcup \Delta_{1}$. By direct calculations, for any $I_{C} \in I_{1}\left(X_{+}[1]_{0},\left[H_{Q}\right]\right)$, we have

$$
\operatorname{Ext}_{X_{+}[1]_{0}}^{1}\left(I_{C}, I_{C}\right) \cong \mathbb{C}, \quad \operatorname{Ext}_{X_{+}[1]_{0}}^{3}\left(I_{C}, I_{C}\right) \cong \mathbb{C}^{2}, \quad \operatorname{Ext}_{X_{+}[1]_{0}}^{i}\left(I_{C}, I_{C}\right)=0, i=2,4 .
$$

Conditions in Definition 5.4 are satisfied, and the relative $D T_{4}$ virtual cycle is the usual fundamental class of the moduli space, i.e.

$$
\left[I_{1}\left(X_{+}[1]_{0},\left[H_{Q}\right]\right)\right] \in H_{2}\left(I_{1}\left(X_{+}[1]_{0},\left[H_{Q}\right]\right), \mathbb{Z}\right) .
$$

We give a gluing formula of relative $D T_{4}$ invariants for the above example.

Example 4.3. In Example 4.2(ii), we consider $X_{0}=X_{+} \cup_{Y} X_{-}$and its smoothing $X_{t}=Q \times \mathbb{T}^{2}$, where $X_{ \pm} \cong Q \times \mathbb{P}^{1}$ and $Y=(Q \times 0) \sqcup(Q \times \infty)$. The moduli space $I_{1}\left(X_{ \pm}[1]_{0},\left[H_{Q}\right]\right)$ of relative ideal sheaves of curves satisfies

$$
I_{1}\left(X_{ \pm}[1]_{0},\left[H_{Q}\right]\right) \cong \bigsqcup_{2875} \mathbb{P}^{1}
$$

The relative $D T_{4}$ virtual cycle is the usual fundamental class of $I_{1}\left(X_{ \pm}[1]_{0},\left[H_{Q}\right]\right)$. Meanwhile, the moduli space $I_{1}\left(X_{t},\left[H_{Q}\right]\right)$ of ideal sheaves of curves in $X_{t}=Q \times \mathbb{T}^{2}$ with curve class $\left[H_{Q}\right]$ satisfies

$$
I_{1}\left(X_{t},\left[H_{Q}\right]\right) \cong \bigsqcup_{2875} \mathbb{T}^{2},
$$

and its $D T_{4}$ virtual cycle is the usual fundamental class of $I_{1}\left(X_{t},\left[H_{Q}\right]\right)$ [13. Under the homologous relation $X_{0} \sim X_{t}$, we have $\mathbb{P}^{1} \cup_{\{0, \infty\}} \mathbb{P}^{1} \sim \mathbb{T}^{2}$. Then the gluing formula is expressed by pairing a $\mu$-map with these cycles (see Theorem 5.12 for such a formula). 
Example 4.4 (Generic quintic in $\left.\mathbb{P}^{4}\right)$. We take $X_{+}=\mathbb{P}^{4}$ which contains a smooth generic quintic 3 -fold $Y=Q$ as its anti-canonical divisor, and then $H_{2}\left(X_{+}, \mathbb{Z}\right) \cong$ $H_{2}(Q, \mathbb{Z}) \cong \mathbb{Z}$. We consider the primitive curve class $[H] \in H_{2}\left(X_{+}, \mathbb{Z}\right)$, and ideal sheaves of curves representing this class have Chern character $c=(1,0,0$, $\left.-P D([H]), \frac{3}{2}\right)$. We denote their moduli space by $I_{\frac{3}{2}}\left(X_{+},[H]\right)(\cong G r(2,5))$. The generic quintic $Q$ contains 2875 rigid degree 1 rational curves, and $I_{\frac{3}{2}}(X,[H])$ contains a finite subset $S$ with 2875 points. $I_{\frac{3}{2}}(X,[H]) \backslash S$ has a well-defined restriction morphism to $\operatorname{Hilb}^{5}(Q)$. To extend the morphism across those 2875 points, we introduce Li-Wu's expanded pair.

We denote $X_{+}[1]_{0}=X_{+} \cup \Delta_{1}, Y[1]_{0}(\cong Q) \subseteq \Delta_{1}$, where $\Delta_{1} \cong \mathbb{P}\left(\left.\mathcal{O}_{Q} \oplus \mathcal{O}_{\mathbb{P}^{4}}(5)\right|_{Q}\right)$, and consider the moduli space $I_{\frac{3}{2}}\left(X_{+}[1]_{0},[H]\right)$ of relative ideal sheaves of curves (normal to the divisor $Q$ ). Geometrically, it is the blowup of $I_{\frac{3}{2}}\left(X_{+},[H]\right.$ ) along those 2875 points, i.e.

$$
I_{\frac{3}{2}}\left(X_{+}[1]_{0},[H]\right) \cong B l_{S}(G r(2,5)),
$$

and each exceptional divisor corresponds to a $\operatorname{Hilb}^{5}\left(\mathbb{P}^{1}\right)\left(\cong \mathbb{P}^{5}\right)$ for each $\mathbb{P}^{1} \subseteq Q$. We then have a restriction morphism

$$
\begin{gathered}
I_{\frac{3}{2}}\left(X_{+}[1]_{0},[H]\right) \rightarrow \operatorname{Hilb}^{5}\left(Y[1]_{0}\right), \\
\left.I_{C} \mapsto I_{C}\right|_{Y[1]_{0}},
\end{gathered}
$$

which is injective with smooth image. By direct calculations, we have

$$
\operatorname{Ext}_{X_{+}[1]_{0}}^{1}\left(I_{C}, I_{C}\right) \cong \mathbb{C}^{6}, \quad \operatorname{Ext}_{X_{+}[1]_{0}}^{2}\left(I_{C}, I_{C}\right) \cong \mathbb{C}^{9}, \quad \operatorname{Ext}_{X_{+}[1]_{0}}^{3}\left(I_{C}, I_{C}\right)=0,
$$

and a long exact sequence

$$
\begin{aligned}
& 0 \rightarrow \operatorname{Ext}_{X_{+}[1]_{0}}^{1}\left(I_{C}, I_{C}\right) \rightarrow \operatorname{Ext}_{Y[1]_{0}}^{1}\left(\left.I_{C}\right|_{Y},\left.I_{C}\right|_{Y}\right) \rightarrow \operatorname{Ext}_{X_{+}[1]_{0}}^{2}\left(I_{C}, I_{C}\right)^{*} \\
\rightarrow & \operatorname{Ext}_{X_{+}[1]_{0}}^{2}\left(I_{C}, I_{C}\right) \rightarrow \operatorname{Ext}_{Y[1]_{0}}^{1}\left(\left.I_{C}\right|_{Y},\left.I_{C}\right|_{Y}\right)^{*} \rightarrow \operatorname{Ext}_{X_{+}[1]_{0}}^{1}\left(I_{C}, I_{C}\right)^{*} \rightarrow 0 .
\end{aligned}
$$

As $\left.I_{C}\right|_{Y} \in \operatorname{Hilb}^{5}\left(Y[1]_{0}\right)$ is a smooth point with $\operatorname{Ext}_{Y[1]_{0}}^{1}\left(\left.I_{C}\right|_{Y},\left.I_{C}\right|_{Y}\right) \cong \mathbb{C}^{15}$, conditions in Definition 5.4 are satisfied. The relative $D T_{4}$ virtual cycle is the usual fundamental class of the moduli space $I_{\frac{3}{2}}\left(X_{+}[1]_{0},[H]\right) \cong B l_{S}(G r(2,5))$.

We adapt Li-Wu's expanded degenerations to torsion sheaves and consider the following extension of Example 4.2

Example 4.5 (Relative $\left.D T_{4} / D T_{3}\right)$. Let $X_{+}=Y_{1} \times \mathbb{P}^{1}$ which contains $Y=\left(Y_{1} \times 0\right) \sqcup$ $\left(Y_{1} \times \infty\right)$ as an anti-canonical divisor, where $Y_{1}$ is a compact Calabi-Yau 3-fold. We denote $\mathfrak{M}_{c}\left(Y_{1}\right)$ to be a Gieseker moduli space of torsion-free semi-stable sheaves on $Y_{1}$ with Chern character $c \in H^{\text {even }}\left(Y_{1}, \mathbb{Q}\right.$ ) (we assume there is no strictly semi-stable sheaf), and denote $\mathfrak{M}_{c}\left(X_{+}\right)$to be the moduli space of sheaves on $X_{+}$which are the push-forward of stable sheaves in $\mathfrak{M}_{c}\left(Y_{1} \times t\right)$ for some $t\left(\mathfrak{M}_{c}\left(X_{+}\right) \cong \mathfrak{M}_{c}\left(Y_{1}\right) \times \mathbb{P}^{1}\right)$. Let $\iota: Y_{1} \times t \hookrightarrow X_{+}$be the natural embedding. For any stable sheaf $\mathcal{F} \in \mathfrak{M}_{c}\left(Y_{1}\right)$, as in Lemma 6.4 of [13], we have

$$
\begin{gathered}
\operatorname{Ext}_{X_{+}}^{1}\left(\iota_{*} \mathcal{F}, \iota_{*} \mathcal{F}\right) \cong \operatorname{Ext}_{Y_{1}}^{1}(\mathcal{F}, \mathcal{F}) \oplus \operatorname{Ext}_{Y_{1}}^{0}(\mathcal{F}, \mathcal{F}), \\
\operatorname{Ext}_{X_{+}}^{2}\left(\iota_{*} \mathcal{F}, \iota_{*} \mathcal{F}\right) \cong \operatorname{Ext}_{Y_{1}}^{2}(\mathcal{F}, \mathcal{F}) \oplus \operatorname{Ext}_{Y_{1}}^{2}(\mathcal{F}, \mathcal{F})^{*} .
\end{gathered}
$$

Furthermore, under the above identifications, a Kuranishi map

$$
\kappa_{\iota_{*}} \mathcal{F}: \operatorname{Ext}_{X_{+}}^{1}\left(\iota_{*} \mathcal{F}, \iota_{*} \mathcal{F}\right) \rightarrow \operatorname{Ext}_{X_{+}}^{2}\left(\iota_{*} \mathcal{F}, \iota_{*} \mathcal{F}\right)
$$


satisfies

$$
\kappa_{\iota_{*}} \mathcal{F}(a, b)=\left(\kappa_{\mathcal{F}}(a), 0\right),
$$

for some Kuranishi map $\kappa_{\mathcal{F}}: \operatorname{Ext}_{Y_{1}}^{1}(\mathcal{F}, \mathcal{F}) \rightarrow \operatorname{Ext}_{Y_{1}}^{2}(\mathcal{F}, \mathcal{F})$ of $\mathfrak{M}_{c}\left(Y_{1}\right)$ at $\mathcal{F}$.

To have a well-defined restriction map, we introduce $X_{+}[1]_{0}=\Delta_{-1} \cup X_{+} \cup \Delta_{1}$, where $\Delta_{ \pm 1} \cong Y_{1} \times \mathbb{P}^{1}$, and consider $\mathfrak{M}_{c}\left(X_{+}[1]_{0}\right)$ to be the union of $\mathfrak{M}_{c}\left(Y_{1}\right) \times \mathbb{C}^{*}$ with the $\mathbb{C}^{*}$-equivalence classes of $\left(\mathfrak{M}_{c}\left(Y_{1} \times 0\right) \times \mathbb{C}^{*}\right) \sqcup\left(\mathfrak{M}_{c}\left(Y_{1} \times \infty\right) \times \mathbb{C}^{*}\right)$, i.e.

$$
\mathfrak{M}_{c}\left(X_{+}[1]_{0}\right) \cong \mathfrak{M}_{c}\left(Y_{1}\right) \times \mathbb{P}^{1} \text {. }
$$

As the supports are disjoint, we have $\mathcal{T}$ or ${ }^{i \geq 1}\left(\iota_{*} \mathcal{F}, \mathcal{O}_{Y}\right)=0$, which implies a long exact sequence similar to the one in Lemma 5.3. and get a canonical isomorphism $\operatorname{Ext}_{X_{+}[1]_{0}}^{2}\left(\iota_{*} \mathcal{F}, \iota_{*} \mathcal{F}\right) \cong \operatorname{Ext}_{X_{+}[1]_{0}}^{2}\left(\iota_{*} \mathcal{F}, \iota_{*} \mathcal{F}\right)^{*}$ as (10). In this specific case, there exists a canonical maximal isotropic subspace $\operatorname{Ext}_{Y_{1}}^{2}(\mathcal{F}, \mathcal{F}) \subseteq \operatorname{Ext}_{X_{+}[1]_{0}}^{2}\left(\iota_{*} \mathcal{F}, \iota_{*} \mathcal{F}\right)$ (66), and $\operatorname{Ext}_{Y_{1}}^{2}(\mathcal{F}, \mathcal{F})$ 's form a sheaf over $\mathfrak{M}_{c}\left(X_{+}[1]_{0}\right)$; thus Conjectures 5.8 and 5.9 hold. Analogous to Theorem 6.5 of [13], the relative $D T_{4}$ virtual cycle satisfies

$$
\left[\mathfrak{M}_{c}^{r e l}\left(X_{+}[1]_{0}\right)\right]^{v i r}=D T_{3}\left(\mathfrak{M}_{c}\left(Y_{1}\right)\right) \cdot\left[\mathbb{P}^{1}\right] \in H_{2}\left(\mathfrak{M}_{c}\left(X_{+}[1]_{0}\right), \mathbb{Z}\right),
$$

where $D T_{3}\left(\mathfrak{M}_{c}\left(Y_{1}\right)\right)$ is the Donaldson-Thomas invariant defined by Thomas [54].

\section{Appendix ON RELATIVE $D T_{4}$ INVARIANTS FOR IDEAL SHEAVES AND GLUING FORMULAS}

5.1. Li-Wu's good degeneration of Hilbert schemes. In this subsection, we recall some basic notions and facts of Li-Wu's good degeneration of Hilbert schemes. The precise definitions are left to their papers [40, [57].

The stack of expanded degenerations. We first introduce the stack of expanded degenerations for pairs $\left(X_{ \pm}, Y\right)$. We replace a pair $\left(X_{+}, Y\right)$ by expanded pairs of length $n,\left(X_{+}[n]_{0}, Y[n]_{0}\right)$, i.e.

$$
X_{+}[n]_{0}=X_{+} \cup \Delta_{1} \cup \cdots \cup \Delta_{n},
$$

which is a chain of smooth irreducible components intersecting transversally with $\Delta_{i}$ to be the $i^{\text {th }}$ copy of $\Delta \triangleq \mathbb{P}\left(\mathcal{N}_{Y / X_{+}} \oplus \mathcal{O}_{Y}\right) . \Delta$ is a $\mathbb{P}^{1}$ bundle over $Y$ with two canonical divisors $Y_{ \pm}$such that $\mathcal{N}_{Y_{+} / X_{+}} \cong \mathcal{N}_{Y / X_{+}}$and $\mathcal{N}_{Y_{-} / X_{+}} \cong \mathcal{N}_{Y / X_{+}}^{*}$. We denote $Y[n]_{0}=Y_{+}$to be the divisor in the last component $\Delta_{n}$. In fact, we can consider families of expanded pairs, $\left(X_{+}[n], Y[n]\right)$ over affine space $\mathbb{A}^{n}$ such that over $0 \in \mathbb{A}^{n}$ it coincides with $\left(X_{+}[n]_{0}, Y[n]_{0}\right)$. Then there exists a pair of Artin stacks $\left(\mathfrak{X}_{+}, \mathfrak{Y}\right)$ as the direct limit of stack quotients of $\left(X_{+}[n], Y[n]\right)$ by certain group actions. The projection of $\left(X_{+}[n], Y[n]\right)$ to the affine space $\mathbb{A}^{n}$ induces a morphism $\mathfrak{Y} \subseteq \mathfrak{X}_{+} \rightarrow \mathfrak{A}_{\diamond}$, where $\mathfrak{A}_{\diamond}$ is the direct limit of some stack quotients of the affine space $\mathbb{A}^{n+1}$.

To formulate the gluing formula, we also need to replace the family $\mathcal{X} \rightarrow C$ by its expanded degeneration $\mathfrak{X} \rightarrow \mathfrak{C}$, where $\mathfrak{X}$ is the direct limit of stack quotients of $X[n]$ and $X[n]$ is a family over $C[n] \triangleq C \times_{\mathbb{A}^{1}} \mathbb{A}^{n+1}, \mathfrak{C} \triangleq C \times_{\mathbb{A}^{1}} \mathfrak{A}$ and $\mathfrak{A}$ is another stack quotient of the affine space $\mathbb{A}^{n+1}$. A smooth chart of $\mathfrak{X}_{0} \triangleq \mathfrak{X} \times_{C} 0$ is

$$
X[n]_{0}=X_{+} \cup \Delta_{1} \cup \cdots \cup \Delta_{n} \cup X_{-},
$$

which is a chain of smooth irreducible components intersecting transversally with $\Delta_{i}$ to be the $i^{\text {th }}$ copy of $\Delta \triangleq \mathbb{P}\left(\mathcal{N}_{Y / X_{+}} \oplus \mathcal{O}_{Y}\right) \cong \mathbb{P}\left(\mathcal{N}_{Y / X_{-}} \oplus \mathcal{O}_{Y}\right)$. We also denote $\Delta_{0}=X_{+}, \Delta_{n+1}=X_{-}$. 
If we consider $X[n]_{0}$ as the gluing of $\left(X_{ \pm}[n]_{0}, Y[n]_{0}\right)$, we need to specify one of its divisors in some $\Delta_{i}$. This is called node-marking, and there exists an Artin stack $\mathfrak{X}_{0}^{\dagger}$ which is the collection of families in $\mathfrak{X}_{0}$ with node-markings. One can construct a stack $\mathfrak{C}_{0}^{\dagger}$ and an arrow $\mathfrak{C}_{0}^{\dagger} \rightarrow \mathfrak{C}$ that fits into a Cartesian product

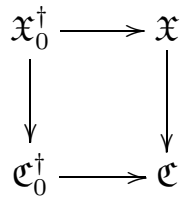

By Proposition 2.13 of [40, there exists a canonical isomorphism $\mathfrak{C}_{0}^{\dagger} \cong \mathfrak{A}_{\diamond}$.

To fix Hilbert polynomials of ideal sheaves over $X[n]_{0}$ and decompose them into ideal sheaves of fixed Hilbert polynomials on $\left(X_{ \pm}[n]_{0}, Y[n]_{0}\right)$, we introduce

$$
\Lambda_{P}^{s p l} \triangleq\left\{\delta=\left(\delta_{ \pm}, \delta_{0}\right) \mid \delta_{+}+\delta_{-}-\delta_{0}=P\right\}
$$

where $\delta_{ \pm}, \delta_{0}, P$ are polynomials in $\mathcal{A} \triangleq \mathcal{A}^{*} \cup\{0\}$, and $\mathcal{A}^{*}$ is the set of $\mathbb{Q}$-coefficient polynomials whose leading terms are of the form $a_{r} \frac{k^{r}}{r !}$ with $a_{r} \in \mathbb{Z}_{+}$.

We define the stack $\mathfrak{X}_{0}^{\dagger, \delta}$ whose closed points are $\left(X[n]_{0}, Y_{k}, w\right)$, where $w$ is a function such that

$$
w\left(\Delta_{[0, k-1]}\right)=\delta_{-}, \quad w\left(\Delta_{[k, n+1]}\right)=\delta_{+}, \quad w\left(Y_{k}\right)=\delta_{0} .
$$

We similarly assign functions $w_{ \pm}$to $\left(X_{ \pm}[n]_{0}, Y[n]_{0}\right)$ with

$$
w_{ \pm}\left(\Delta_{[0, n]}\right)=\delta_{ \pm}, \quad w_{ \pm}\left(Y[n]_{0}\right)=\delta_{0}
$$

and define stacks $\mathfrak{X}_{ \pm}^{\delta_{ \pm}, \delta_{0}}$. Then there exist stacks $\mathfrak{A}_{\diamond}^{\delta_{ \pm}, \delta_{0}}$ so that we have the Cartesian product

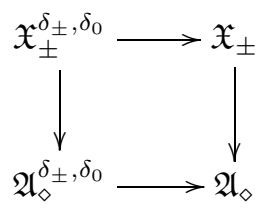

By gluing two components, we obtain the following commutative diagram:

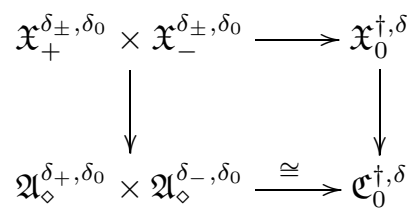

We denote $\mathfrak{C}_{0}^{\dagger, P}=\bigsqcup_{\delta \in \Lambda_{P}^{s p l}} \mathfrak{C}_{0}^{\dagger, \delta}$ and then have a natural morphism $\Phi_{\delta}: \mathfrak{C}_{0}^{\dagger, \delta} \rightarrow \mathfrak{C}^{\mathrm{r} P}$ as the composition of the imbedding $\mathfrak{C}_{0}^{\dagger, \delta} \rightarrow \mathfrak{C}_{0}^{\dagger, P}$ with forgetful map $\mathfrak{C}_{0}^{\dagger, P} \rightarrow \mathfrak{C}^{P}$. 
Lemma 5.1 (Li-Wu, Proposition 2.19, 40). There are canonical line bundles with sections $\left(L_{\delta}, s_{\delta}\right)$ on $\mathfrak{C}^{P}$ indexed by $\delta \in \Lambda_{P}^{\text {spl }}$, such that

(1) letting $t$ be the standard coordinate function on $\mathbb{A}^{1}$ and $\pi: \mathfrak{C}^{P} \rightarrow \mathbb{A}^{1}$ be the tautological projection, then

$$
\bigotimes_{\delta \in \Lambda_{P}^{s p l}} L_{\delta} \cong \mathcal{O}_{\mathfrak{C}^{P}}, \quad \prod_{\delta \in \Lambda_{P}^{s p l}} s_{\delta}=\pi^{*} t
$$

(2) $\Phi_{\delta}$ factors through $s_{\delta}^{-1}(0) \subseteq \mathfrak{C}^{P}$ and there exists an isomorphism $s_{\delta}^{-1}(0) \cong$ $\mathfrak{C}_{0}^{\dagger, \delta}$.

This states that $\mathfrak{C}_{0}^{P} \subseteq \mathfrak{C}^{P}$ is a complete intersection substack with $\bigsqcup_{\delta \in \Lambda_{P}^{s p l}} \mathfrak{C}_{0}^{\dagger, \delta}$ as its normalization.

Moduli stacks of stable ideal sheaves. By Theorem 4.14 of [40], there exists a Deligne-Mumford stack $\mathfrak{I}_{\mathfrak{X} / \mathfrak{C}}^{P}$ which is of finite type, separated and proper over $C$. It is a good degeneration of the Hilbert scheme of subschemes of $X / C$ with fixed Hilbert polynomial $P$ in the sense that

$$
\mathfrak{I}_{\mathfrak{X} / \mathfrak{C}}^{P} \times_{C} t \cong \operatorname{Hilb}^{P}\left(X_{t}\right), \quad t \neq 0,
$$

and the central fiber $\mathfrak{I}_{\mathfrak{X}_{0} / \mathfrak{C}_{0}}^{P} \triangleq \mathfrak{I}_{\mathfrak{X} / \mathfrak{C}}^{P} \times_{C} 0$ has a good obstruction theory. We recall that any closed point of $\mathfrak{I}_{\mathfrak{X}_{0} / \mathfrak{c}_{0}}^{P}$ is an ideal sheaf $I_{Z}$ in some $X[n]_{0}$ such that

(1) $\mathcal{O}_{Z}$ is normal to all $Y_{i} \subseteq X[n]_{0}$, i.e. $\operatorname{Tor}_{\mathcal{O}_{X[n]_{0}}}^{1}\left(\mathcal{O}_{Z}, \mathcal{O}_{Y_{i}}\right)=0$.

(2) $\operatorname{Aut}_{\mathfrak{X}}\left(I_{Z}\right)$ is finite.

(3) The Hilbert polynomial of $\mathcal{O}_{Z}$ is $P$.

We define

$$
\mathfrak{I}_{\mathfrak{X}_{0}^{\dagger} / \mathfrak{C}_{0}^{\dagger}}^{\delta} \triangleq \mathfrak{I}_{\mathfrak{X} / \mathfrak{C}^{P}}^{P} \times \mathfrak{C}^{P} \mathfrak{C}_{0}^{\dagger, \delta}
$$

It parameterizes ideal sheaves $I_{Z}$ 's on $X[n]_{0}$ with a node-marking $Y_{k} \subseteq X[n]_{0}$ so that the Hilbert polynomials of $\mathcal{O}_{Z}$ restricted to $\bigcup_{i<k} \Delta_{i}$, to $\bigcup_{i \geq k} \Delta_{i}$ and to $Y_{k}$ are $\delta_{-}, \delta_{+}$and $\delta_{0}$ respectively.

We can similarly define the moduli stack of stable relative ideal sheaves for $\mathfrak{Y} \subseteq \mathfrak{X}_{ \pm}$with a pair of Hilbert polynomials $\left(\delta_{ \pm}, \delta_{0}\right)$, denoted by $\mathfrak{I}_{\mathfrak{X}_{ \pm} / \delta_{\diamond}}^{\delta_{ \pm}, \mathfrak{A}_{\diamond}}$, which are of finite type, separated and proper Deligne-Mumford stacks (Theorem 4.15 of [40]). The relations between $\mathfrak{I}_{\mathfrak{X} / \mathfrak{C}}^{P}, \mathfrak{I}_{\mathfrak{X}_{0}^{\dagger} / \mathfrak{C}_{0}^{\dagger}}^{\delta}$ and $\mathfrak{I}_{\mathfrak{X}_{ \pm} / \mathfrak{A}_{\diamond}}^{\delta_{ \pm}, \delta_{0}}$ are described as follows.

Lemma 5.2 (Li-Wu, Theorem 5.27, 40]). (1) There exist natural restriction morphisms $\mathfrak{I}_{\mathfrak{X}_{ \pm} / \delta_{0}}^{\delta_{ \pm}, \mathcal{A}_{\diamond}} \rightarrow H i l b_{Y}^{\delta_{0}}$, where Hilb $\delta_{Y}^{\delta_{0}}$ is the Hilbert scheme on $Y$ with fixed Hilbert polynomial $\delta_{0}$, and an isomorphism

$$
\mathfrak{I}_{\mathfrak{X}_{-} / \mathfrak{A}_{\diamond}}^{\delta_{-}, \delta_{0}} \times_{H_{i l b} b_{Y}^{\delta_{0}}} \mathfrak{I}_{\mathfrak{X}_{+} / \mathfrak{A}_{\diamond}}^{\delta_{+}, \delta_{0}} \rightarrow \mathfrak{I}_{\mathfrak{X}_{0}^{\dagger} / \mathfrak{C}_{0}^{\dagger}}^{\delta} \cdot
$$

(2) Let $\left(L_{\delta}, s_{\delta}\right)$ be as in Lemma 5.1 and $\pi_{P}: \mathfrak{I}_{\mathfrak{X} / \mathfrak{C}}^{P} \rightarrow \mathfrak{C}^{P}$ be the natural projection. Then

$$
\bigotimes_{\delta \in \Lambda_{P}^{s p l}} \pi_{P}^{*} L_{\delta} \cong \mathcal{O}_{\mathfrak{\Im}_{\mathfrak{X} / \mathfrak{C}}^{P}}, \quad \prod_{\delta \in \Lambda_{P}^{s p l}} \pi_{P}^{*} s_{\delta}=\pi_{P}^{*} \pi^{*} t .
$$

As closed substacks of $\mathfrak{I}_{\mathfrak{X} / \mathfrak{C}}^{P}$, we have $\mathfrak{I}_{\mathfrak{X}_{0}^{\dagger} / \mathfrak{C}_{0}^{\dagger}}^{\delta} \cong\left(\pi_{P}^{*} s_{\delta}=0\right)$. 
5.2. Relative $D T_{4}$ virtual cycles. We study obstruction theories of DeligneMumford stacks $\mathfrak{I}_{\mathfrak{X}_{0}^{\dagger} / \mathfrak{C}_{0}^{\dagger}}^{\delta}$ and $\mathfrak{I}_{\mathfrak{X}_{ \pm} / \mathfrak{A}_{\diamond}}^{\delta_{ \pm}, \delta_{0}}$.

Lemma 5.3. We take a closed point $\left[I_{Z}\right] \in \mathfrak{I}_{\mathfrak{X}_{+} / \mathfrak{A}_{\diamond}}^{\delta_{+}, \delta_{0}}$ with $Z \subseteq X_{+}[n]_{0}$. Then for $Y=Y[n]_{0}$, we have a short exact sequence

$$
0 \rightarrow I_{Z} \otimes \mathcal{O}_{X_{+}[n]_{0}}(-Y) \rightarrow I_{Z} \rightarrow I_{Z} \otimes \mathcal{O}_{Y} \rightarrow 0
$$

and a long exact sequence

$$
\begin{aligned}
& \quad \cdots \rightarrow \operatorname{Ext}^{1}\left(I_{Z}, I_{Z} \otimes \mathcal{O}_{X_{+}[n]_{0}}(-Y)\right) \rightarrow \operatorname{Ext}^{1}\left(I_{Z}, I_{Z}\right) \rightarrow \operatorname{Ext}_{Y}^{1}\left(I_{Z} \otimes \mathcal{O}_{Y}, I_{Z} \otimes \mathcal{O}_{Y}\right) \\
& \quad \rightarrow \operatorname{Ext}^{2}\left(I_{Z}, I_{Z} \otimes \mathcal{O}_{X_{+}[n]_{0}}(-Y)\right) \rightarrow \operatorname{Ext}^{2}\left(I_{Z}, I_{Z}\right) \rightarrow \operatorname{Ext}_{Y}^{2}\left(I_{Z} \otimes \mathcal{O}_{Y}, I_{Z} \otimes \mathcal{O}_{Y}\right) \\
& \rightarrow \operatorname{Ext}^{3}\left(I_{Z}, I_{Z} \otimes \mathcal{O}_{X_{+}[n]_{0}}(-Y)\right) \rightarrow \operatorname{Ext}^{3}\left(I_{Z}, I_{Z}\right) \rightarrow \cdots .
\end{aligned}
$$

Proof. We tensor $0 \rightarrow I_{Z} \rightarrow \mathcal{O}_{X_{+}[n]_{0}} \rightarrow \mathcal{O}_{Z} \rightarrow 0$ with $\mathcal{O}_{Y}$ and get

$$
\mathcal{T}_{\text {or }} \mathcal{O}_{X_{+}[n]_{0}}^{i+1}\left(\mathcal{O}_{Z}, \mathcal{O}_{Y}\right) \cong \mathcal{T}_{\text {or }}^{i}{ }_{\mathcal{O}_{X_{+}[n]_{0}}}\left(I_{Z}, \mathcal{O}_{Y}\right), \quad i \geq 1
$$

Applying the tensor product with $\mathcal{O}_{Z}$ to $0 \rightarrow \mathcal{O}_{X_{+}[n]_{0}}(-Y) \rightarrow \mathcal{O}_{X_{+}[n]_{0}} \rightarrow \mathcal{O}_{Y} \rightarrow 0$, we get

$$
\mathcal{T}_{\text {or }_{\mathcal{O}}}^{i \geq 2}{ }_{X^{[n]_{0}}}\left(\mathcal{O}_{Z}, \mathcal{O}_{Y}\right)=0 .
$$

These ensure that we have the short exact sequence (7) after tensoring

$$
0 \rightarrow \mathcal{O}_{X_{+}[n]_{0}}(-Y) \rightarrow \mathcal{O}_{X_{+}[n]_{0}} \rightarrow \mathcal{O}_{Y} \rightarrow 0
$$

with $I_{Z}$. We then take $\operatorname{Hom}\left(I_{Z}, \cdot\right)$ to (7) and are left to show that

$$
\operatorname{Ext}^{*}\left(I_{Z}, I_{Z} \otimes \mathcal{O}_{Y}\right) \cong \operatorname{Ext}_{Y}^{*}\left(I_{Z} \otimes \mathcal{O}_{Y}, I_{Z} \otimes \mathcal{O}_{Y}\right) .
$$

We have a spectral sequence

$$
H^{*}\left(X_{+}[n]_{0}, \mathcal{E} x t^{*}\left(I_{Z}, I_{Z} \otimes \mathcal{O}_{Y}\right)\right) \Rightarrow \operatorname{Ext}^{*}\left(I_{Z}, I_{Z} \otimes \mathcal{O}_{Y}\right) .
$$

By Corollary 2.9 [57, we can take a finite length locally free resolution $E^{\bullet} \rightarrow I_{Z} \rightarrow$ 0 . Then

$$
\begin{aligned}
& H^{*}\left(X_{+}[n]_{0}, \mathcal{E x t}^{*}\left(I_{Z}, I_{Z} \otimes \mathcal{O}_{Y}\right)\right) \cong H^{*}\left(X_{+}[n]_{0}, \mathcal{E} x t^{*}\left(\mathcal{O}_{X_{+}[n]_{0}}, \mathcal{O}_{Y}\right) \otimes \operatorname{End}\left(E^{\bullet}\right)\right) \\
\cong & H^{*}\left(X_{+}[n]_{0}, \operatorname{End}\left(E^{\bullet}\right) \otimes \mathcal{O}_{Y}\right) \cong H^{*}\left(X_{+}[n]_{0}, \iota_{*} \operatorname{End}\left(\left.E^{\bullet}\right|_{Y}\right)\right) \cong \operatorname{Ext}_{Y}^{*}\left(\left.E^{\bullet}\right|_{Y},\left.E^{\bullet}\right|_{Y}\right),
\end{aligned}
$$
where $\iota: Y \hookrightarrow X_{+}[n]_{0}$ is the closed imbedding. By (8), (9), we have

$$
\mathcal{T}_{\text {or }}^{i \geq 1} \mathcal{O}_{X_{+}[n]_{0}}\left(I_{Z}, \mathcal{O}_{Y}\right)=0
$$

which implies that $\left.\left.E^{\bullet}\right|_{Y} \rightarrow I_{Z}\right|_{Y} \rightarrow 0$ is still a resolution. Thus $\operatorname{Ext}_{Y}^{*}\left(\left.I_{Z}\right|_{Y},\left.I_{Z}\right|_{Y}\right)$ $\cong \operatorname{Ext}^{*}\left(I_{Z}, I_{Z} \otimes \mathcal{O}_{Y}\right)$.

The above long exact sequence is the ideal sheaf version of the long exact sequence in Lemma 3.3. We consider the following extension of virtual cycles for bundles (Cases I-III) to ideal sheaf cases.

Definition 5.4. Let $Y$ be an anti-canonical divisor of a complex projective 4-fold $X_{+}$, and

$$
r: \mathfrak{I}_{\mathfrak{X}_{+} / \mathfrak{A}_{\diamond}}^{\delta_{+} \delta_{0}} \rightarrow H i l b_{Y}^{\delta_{0}}
$$

be Li-Wu's restriction morphism in Lemma 5.2 We assume $\mathfrak{I}_{\mathfrak{X}_{+} / \mathfrak{A}_{\diamond}}^{\delta_{+}, \delta_{0}}$ is a smooth moduli scheme (all Kuranishi maps vanish). 
The relative $D T_{4}$ virtual cycle for $\mathfrak{I}_{\mathfrak{X}_{+} / \mathfrak{A}_{\diamond}}^{\delta_{+}, \delta_{0}}$ is its usual fundamental class provided that any one of the following conditions holds:

(1) $r$ is surjective between smooth moduli spaces and the obstruction bundle $\operatorname{Ob}\left(\mathfrak{I}_{\mathfrak{X}_{+} / \mathfrak{A}_{\diamond}}^{\delta_{+}, \delta_{0}}\right)=0$,

(2) $r$ is injective between smooth moduli spaces (at least when restricted to a neighbourhood $U\left(r\left(\mathfrak{I}_{\mathfrak{X}_{+} / \mathfrak{A}_{\diamond}}^{\delta_{+}, \delta_{0}}\right)\right)$ of $r\left(\mathfrak{I}_{\mathfrak{X}_{+} / \mathfrak{A}_{\diamond}}^{\delta_{+}, \delta_{0}}\right)$ in $\left.H i l b_{Y}^{\delta_{0}}\right)$, and $r k\left(O b\left(\mathfrak{I}_{\mathfrak{X}_{+} / \mathfrak{A}_{\diamond}}^{\delta_{+}, \delta_{0}}\right)\right)=$ $\operatorname{codim}\left(\mathfrak{I}_{\mathfrak{X}_{+} / \mathfrak{A}_{\diamond}}^{\delta_{\beta_{0}}, \delta_{0}}, U\left(r\left(\mathfrak{I}_{\mathfrak{X}_{+} / \mathfrak{A}_{\diamond}}^{\delta_{+}, \delta_{0}}\right)\right)\right.$.

A modification by twisting $K_{X_{ \pm}}^{\frac{1}{2}}$. In general, extensions of virtual cycles for bundles (Cases I-III) to ideal sheaves are not straightforward. We study the extension for Case I.

Proposition 5.5. We take a smooth Calabi-Yau 3-fold $Y$ in complex projective 4folds $X_{ \pm}$as their anti-canonical divisors. We assume any $I_{C} \in \operatorname{Hilb}^{\delta_{0}}(Y)$ satisfies $\operatorname{Ext}^{1}\left(I_{C}, I_{C}\right)=0$. Then for any closed point of $\mathfrak{I}_{\mathfrak{X}_{ \pm} / \delta_{\diamond}}^{\delta_{ \pm}, \delta_{0}}$, say $\left[I_{Z_{ \pm}}\right]$with $Z_{ \pm} \subseteq$ $X_{ \pm}[n]_{0}$, we have canonical isomorphisms

$$
\operatorname{Ext}_{X_{ \pm}[n]_{0}}^{i}\left(I_{Z_{ \pm}}, I_{Z_{ \pm}}\right)_{0} \cong \operatorname{Ext}_{X_{ \pm}[n]_{0}}^{4-i}\left(I_{Z_{ \pm}}, I_{Z_{ \pm}}\right)_{0}^{*}, i=1,2 .
$$

Furthermore, under the isomorphism in Lemma 5.2 ,

$$
\mathfrak{I}_{\mathfrak{X}_{0}^{\dagger} / \mathfrak{C}_{0}^{\dagger}}^{\delta} \cong \mathfrak{I}_{\mathfrak{X}_{-} / \mathfrak{A}_{\diamond}}^{\delta_{-}, \delta_{0}} \times_{H_{i l b}}^{\delta_{0}} \mathfrak{I}_{\mathfrak{X}_{+} / \mathfrak{A}_{\diamond}}^{\delta_{+}, \delta_{0}},
$$

where a closed point of $\mathfrak{I}_{\mathfrak{X}_{0}^{\dagger} / \mathfrak{C}_{0}^{\dagger}}^{\delta}$ is written as $I_{Z}=I_{Z_{+}} \cup I_{Z_{-}}$, with $Z \subseteq X\left[n_{+}+n_{-}\right]_{0}$, $Z_{ \pm} \subseteq X_{ \pm}\left[n_{ \pm}\right]_{0}$, we have canonical isomorphisms of trace-free extension groups

$$
\operatorname{Ext}_{X\left[n_{+}+n_{-}\right]_{0}}^{*}\left(I_{Z}, I_{Z}\right)_{0} \cong \operatorname{Ext}_{X_{+}\left[n_{+}\right]_{0}}^{*}\left(I_{Z_{+}}, I_{Z_{+}}\right)_{0} \oplus \operatorname{Ext}_{X_{-}\left[n_{-}\right]_{0}}^{*}\left(I_{Z_{-}}, I_{Z_{-}}\right)_{0},
$$

under which the non-degenerate quadratic forms on $\operatorname{Ext}_{X\left[n_{+}+n_{-}\right]_{0}}^{2}\left(I_{Z}, I_{Z}\right)_{0}$ and Ext $t_{X_{ \pm}[n]_{0}}^{2}\left(I_{Z_{ \pm}}, I_{Z_{ \pm}}\right)$(10) are preserved.

Proof. We apply the trace-free version of Lemma 5.3 to the case when $Y$ is the Cartier divisor associated with the dualizing sheaf of $X_{ \pm}[n]_{0}$ and get canonical isomorphisms by Serre duality.

We take a closed point $I_{Z} \in \mathfrak{I}_{\mathfrak{X}_{0}^{\dagger} / \mathfrak{C}_{0}^{\dagger}}^{\delta}$ with $Z \subseteq X\left[n_{+}+n_{-}\right]_{0}=X_{+}\left[n_{+}\right]_{0} \cup_{Y}$ $X_{-}\left[n_{-}\right]_{0}$ and restrict to get $I_{Z_{ \pm}} \subseteq \mathcal{O}_{X_{ \pm}\left[n_{ \pm}\right]}$. As in [46], [40, we then get an exact triangle

$$
\begin{aligned}
\operatorname{RHom}_{X\left[n_{+}+n_{-}\right]_{0}}\left(I_{Z}, I_{Z}\right)_{0} & \rightarrow \bigoplus \operatorname{RHom}_{X_{ \pm}\left[n_{ \pm}\right]_{0}}\left(\left.I_{Z}\right|_{X_{ \pm}\left[n_{ \pm}\right]_{0}},\left.I_{Z}\right|_{X_{ \pm}\left[n_{ \pm}\right]_{0}}\right)_{0} \\
& \rightarrow \operatorname{RHom}_{Y}\left(\left.I_{Z}\right|_{Y},\left.I_{Z}\right|_{Y}\right)_{0} .
\end{aligned}
$$

We take cohomology and use the assumption to deduce $H^{*}\left(\operatorname{RHom}_{Y}\left(\left.I_{Z}\right|_{Y},\left.I_{Z}\right|_{Y}\right)_{0}\right)$ $=0$.

From Proposition 5.5, one may expect to get a $(-2)$-shifted symplectic structure on $\mathfrak{I}_{\mathfrak{X}_{ \pm} / \delta_{0}}^{\delta_{ \pm}, \mathfrak{A}_{\diamond}}$ if any $I_{C} \in H i l b^{\delta_{0}}(Y)$ satisfies $\operatorname{Ext}^{1}\left(I_{C}, I_{C}\right)=0$. Then as BorisovJoyce did in [7, one expects to use BBJ type local Darboux charts, partition of unity and homotopical algebra to obtain D-orbifolds associated with $\mathfrak{I}_{\mathfrak{X}_{ \pm} / \mathfrak{A}_{\diamond}}^{\delta_{ \pm}}$and $\mathfrak{I}_{\mathfrak{X}_{0}^{\dagger} / \mathfrak{C}_{0}^{\dagger}}^{\delta}$ In particular, analogous to Theorem 2.6, there should exist homology 
classes $\left[\mathfrak{I}_{\mathfrak{X}_{ \pm} / \mathfrak{A}_{\diamond}}^{\delta_{ \pm}, \delta_{0}}\right]^{\text {vir }} \in H_{*}\left(\mathfrak{I}_{\mathfrak{X}_{ \pm} / \mathfrak{A}_{\diamond}}^{\delta_{\delta}, \delta_{0}}, \mathbb{Q}\right),\left[\mathfrak{I}_{\mathfrak{X}_{0}^{\dagger} / \mathfrak{C}_{0}^{\dagger}}^{\delta}\right]^{\text {vir }} \in H_{*}\left(\mathfrak{I}_{\mathfrak{X}_{0}^{\dagger} / \mathfrak{C}_{0}^{\dagger}}^{\delta}, \mathbb{Q}\right)$ if the associated D-orbifolds of $\mathfrak{I}_{\mathfrak{X}_{ \pm} / \mathfrak{A}_{\diamond}}^{\delta_{ \pm}, \delta_{0}}$ and $\mathfrak{I}_{\mathfrak{X}_{0}^{\dagger} / \mathfrak{C}_{0}^{\dagger}}^{\delta}$ are orientable [30].

However, under the assumption that any $I_{C} \in \operatorname{Hilb}^{\delta_{0}}(Y)$ satisfies $\operatorname{Ext}^{1}\left(I_{C}, I_{C}\right)=$ 0, the non-degenerate quadratic forms on $\operatorname{Ext}_{X_{ \pm}[n]_{0}}^{2}\left(I_{Z_{ \pm}}, I_{Z_{ \pm}}\right)$(see Proposition 5.5) don't have to glue together, and the D-orbifold associated with $\mathfrak{I}_{\mathfrak{X}_{+} / \delta^{\prime}}^{\delta_{\mathcal{A}}}$ might not exist (see Example 4.1). For gluing, we discuss the case when there exists a square root $K_{X_{ \pm}}^{\frac{1}{2}}$ of $K_{X_{ \pm}}$with $K_{X_{ \pm}}^{\frac{1}{2}} \otimes K_{X_{ \pm}}^{\frac{1}{2}} \cong K_{X_{ \pm}}$.

Lemma 5.6. Let $X_{+}$be a complex projective 4 -fold with a square root $K_{X_{+}}^{\frac{1}{2}}, Y_{i}$ $(i=1,2)$ be two smooth zero locus of sections of $K_{X_{+}}^{-\frac{1}{2}}$ with $K_{Y_{i}}=0\left(\Leftrightarrow \mathcal{N}_{Y_{i} / X_{+}} \cong\right.$ $\left.\mathcal{O}_{Y_{i}}\right)$ and $Y_{1} \cap Y_{2}=\emptyset$. We take $Y=Y_{1} \sqcup Y_{2}$ which is a smooth anti-canonical divisor of $X_{+}$. Then for any closed point $\left[I_{Z}\right] \in \mathfrak{I}_{\mathfrak{X}_{+} / \mathfrak{A}_{\diamond}}^{\delta_{+}, \delta_{0}}$ with $Z \subseteq X_{+}[n]_{0}$ and $Y[n]_{0}=Y_{1} \sqcup Y_{2}$, we have a short exact sequence

$$
0 \rightarrow I_{Z} \otimes K_{X_{+}[n]_{0}}^{\frac{1}{2}} \rightarrow I_{Z} \rightarrow I_{Z} \otimes \mathcal{O}_{Y_{1}} \rightarrow 0
$$

and a long exact sequence

$$
\begin{aligned}
0 & \rightarrow \operatorname{Ext}_{X_{+}[n]_{0}}^{1}\left(I_{Z}, I_{Z} \otimes K_{X_{+}[n]_{0}}^{\frac{1}{2}}\right)_{0} \rightarrow \operatorname{Ext}_{X_{+}[n]_{0}}^{1}\left(I_{Z}, I_{Z}\right)_{0} \\
& \rightarrow \operatorname{Ext}_{Y_{1}}^{1}\left(I_{Z} \otimes \mathcal{O}_{Y_{1}}, I_{Z} \otimes \mathcal{O}_{Y_{1}}\right)_{0} \\
& \rightarrow \operatorname{Ext}_{X_{+}[n]_{0}}^{2}\left(I_{Z}, I_{Z} \otimes K_{X_{+}[n]_{0}}^{\frac{1}{2}}\right)_{0} \rightarrow \operatorname{Ext}_{X_{+}[n]_{0}}^{2}\left(I_{Z}, I_{Z}\right)_{0} \\
& \rightarrow \operatorname{Ext}_{Y_{1}}^{2}\left(I_{Z} \otimes \mathcal{O}_{Y_{1}}, I_{Z} \otimes \mathcal{O}_{Y_{1}}\right)_{0} \rightarrow \cdots
\end{aligned}
$$

where $K_{X_{+}[n]_{0}}^{\frac{1}{2}}$ is a square root of the dualizing sheaf of $X_{+}[n]_{0}$.

Furthermore, if any $I_{C} \in \operatorname{Hilb}^{\delta_{0}}(Y)$ satisfies Ext $t_{Y}^{1}\left(I_{C}, I_{C}\right)=0$, we have canonical isomorphisms

$$
\begin{gathered}
\phi_{1}: \operatorname{Ext}_{X_{+}[n]_{0}}^{2}\left(I_{Z}, I_{Z} \otimes K_{X_{+}[n]_{0}}\right)_{0} \cong \operatorname{Ext}_{X_{+}[n]_{0}}^{2}\left(I_{Z}, I_{Z} \otimes K_{X_{+}[n]_{0}}^{\frac{1}{2}}\right)_{0}, \\
\phi_{2}: \operatorname{Ext}_{X_{+}[n]_{0}}^{2}\left(I_{Z}, I_{Z} \otimes K_{X_{+}[n]_{0}}^{\frac{1}{2}}\right)_{0} \cong \operatorname{Ext}_{X_{+}[n]_{0}}^{2}\left(I_{Z}, I_{Z}\right)_{0} .
\end{gathered}
$$

Proof. It is similar to the proof of Lemma 5.3. The isomorphism $\phi_{1}$ is derived by tensoring $0 \rightarrow I_{Z} \otimes K_{X_{+}[n]_{0}}^{\frac{1}{2}} \rightarrow I_{Z} \rightarrow I_{Z} \otimes \mathcal{O}_{Y_{1}} \rightarrow 0$ with $K_{X_{+}[n]_{0}}^{\frac{1}{2}}$ and taking the long exact sequence.

The reason for introducing $\operatorname{Ext}_{X_{+}[n]_{0}}^{2}\left(I_{Z}, I_{Z} \otimes K_{X_{+}[n]_{0}}^{\frac{1}{2}}\right)_{0}$ is that Serre duality pairing defines a natural non-degenerate quadratic form on it. If $E x t_{X_{+}[n]_{0}}^{2}\left(I_{Z_{+}}, I_{Z_{+}}\right.$ $\left.\otimes K_{X_{+}[n]_{0}}^{\frac{1}{2}}\right)_{0}$ 's are glued to be a sheaf over the moduli space, then the Serre duality pairing will probably 'glue'. By Proposition 5.5, there is a non-degenerate quadratic form on $\operatorname{Ext}_{X_{+}[n]_{0}}^{2}\left(I_{Z}, I_{Z}\right)_{0}$. We make a comparison between them.

Proposition 5.7. Let $X_{+}$be a complex projective 4-fold with a square root $K_{X_{+}}^{\frac{1}{2}}, Y_{i}$ $(i=1,2)$ be two smooth zero locus of sections of $K_{X_{+}}^{-\frac{1}{2}}$ with $K_{Y_{i}}=0\left(\Leftrightarrow \mathcal{N}_{Y_{i} / X_{+}} \cong\right.$ $\left.\mathcal{O}_{Y_{i}}\right)$ and $Y_{1} \cap Y_{2}=\emptyset$. We take $Y=Y_{1} \sqcup Y_{2}$ which is a smooth anti-canonical divisor of $X_{+}$and assume any $I_{C} \in \operatorname{Hilb}^{\delta_{0}}(Y)$ satisfies $\operatorname{Ext}_{Y}^{1}\left(I_{C}, I_{C}\right)=0$. Then 
for any closed point $\left[I_{Z}\right] \in \mathfrak{I}_{\mathfrak{X}_{+} / \mathfrak{A}_{\diamond}}^{\delta_{+}, \delta_{0}}$ with $Z \subseteq X_{+}[n]_{0}$ and $Y[n]_{0}=Y_{1} \sqcup Y_{2}$, we have a commutative diagram

$$
\begin{aligned}
& \operatorname{Ext}_{X_{+}[n]_{0}}^{2}\left(I_{Z}, I_{Z} \otimes K_{X_{+}[n]_{0}}\right)_{0} \\
& \phi_{1} \downarrow \\
& \underset{\phi_{3}}{\phi_{X}}
\end{aligned}
$$

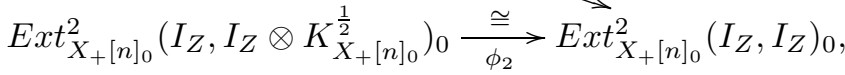

where $\phi_{1}, \phi_{2}$ are defined in Lemma 5.6 and $\phi_{3}$ is the isomorphism induced from the long exact sequence in Lemma 5.3. Furthermore, $\phi_{2}$ is an isometry with respect to the Serre duality pairing on $\operatorname{Ext}_{X_{+}[n]_{0}}^{2}\left(I_{Z}, I_{Z} \otimes K_{X_{+}[n]_{0}}^{\frac{1}{2}}\right)_{0}$ and the quadratic form on $\operatorname{Ext}_{X_{+}[n]_{0}}^{2}\left(I_{Z}, I_{Z}\right)_{0}$ defined in Proposition 5.5 .

Proof. The commutativity is because isomorphisms $\phi_{1}, \phi_{2}$ are pairings with sections in $H^{0}\left(X_{+}[n]_{0}, K_{X_{+}[n]_{0}}^{-\frac{1}{2}}\right)$ corresponding to Cartier divisors $Y_{1}, Y_{2}$, and $\phi_{3}$ is the pairing with a section in $H^{0}\left(X_{+}[n]_{0}, K_{X_{+}[n]_{0}}^{-1}\right)$ corresponding to $Y=Y_{1} \sqcup Y_{2}$. Then it is easy to check that $\phi_{2}$ is an isometry.

Conjecture 5.8. Let $Y$ be a smooth Calabi-Yau 3-fold in complex projective 4folds $X_{ \pm}$as their anti-canonical divisors. We assume any $I_{C} \in \operatorname{Hilb}^{\delta_{0}}(Y)$ satisfies $\operatorname{Ext}^{1}\left(I_{C}, I_{C}\right)=0$. Then there exists a D-orbifold associated with Deligne-Mumford stack $\mathfrak{I}_{\mathfrak{X}_{0}^{\dagger} / \mathfrak{C}_{0}^{\dagger}}$; i.e. they have the same underlying topological structures.

Furthermore, if canonical bundles of $X_{ \pm}$admit square roots $K_{X_{ \pm}}^{\frac{1}{2}}$ and there exist $Y_{i}(i=1,2)$ which are smooth zero locus of sections of $K_{X_{ \pm}}^{-\frac{1}{2}}$ with $K_{Y_{i}}=0$ $\left(\Leftrightarrow \mathcal{N}_{Y_{i} / X_{ \pm}} \cong \mathcal{O}_{Y_{i}}\right)$ such that $Y=Y_{1} \cup Y_{2}, Y_{1} \cap Y_{2}=\emptyset$, then there exist D-orbifolds associated with Deligne-Mumford stacks $\mathfrak{I}_{\mathfrak{X}_{ \pm} / \mathfrak{A}_{\diamond}}^{\delta_{ \pm}, \delta_{0}}$.

Conjecture 5.9. We take a smooth Calabi-Yau 3-fold $Y$ in complex projective 4-folds $X_{ \pm}$as their anti-canonical divisors. We assume any $I_{C} \in H_{i l b} \delta_{0}(Y)$ satisfies $\operatorname{Ext}^{1}\left(I_{C}, I_{C}\right)=0$ (we assume $H_{i l b} \delta_{0}(Y)$ consists of one point without loss of generality). Then we have

$$
\left[\mathfrak{I}_{\mathfrak{X}_{ \pm} / \mathfrak{A}_{\diamond}}^{\delta_{ \pm}, \delta_{0}}\right]^{\text {vir }} \in H_{*}\left(\mathfrak{I}_{\mathfrak{X}_{ \pm} / \mathfrak{A}_{\diamond}}^{\delta_{ \pm}, \delta_{0}}, \mathbb{Q}\right),\left[\mathfrak{I}_{\mathfrak{X}_{0}^{\dagger} / \mathfrak{C}_{0}^{\dagger}}^{\delta}\right]^{\text {vir }} \in H_{*}\left(\mathfrak{I}_{\mathfrak{X}_{0}^{\dagger} / \mathfrak{C}_{0}^{\dagger}}^{\delta}, \mathbb{Q}\right)
$$

if there exist orientable D-orbifolds [30] associated with $\mathfrak{I}_{\mathfrak{X}_{ \pm} / \mathfrak{A}_{\diamond}}^{\delta_{ \pm}, \delta_{0}}$ and $\mathfrak{I}_{\mathfrak{X}_{0}^{\dagger} / \mathfrak{C}_{0}^{\dagger}}^{\delta}$. Furthermore, under the isomorphism

$$
\mathfrak{I}_{\mathfrak{X}_{-} / \mathfrak{A}_{\diamond}}^{\delta_{-}, \delta_{0}} \times{ }_{H_{H i l b}}^{\delta_{0}} \mathfrak{I}_{\mathfrak{X}_{+} / \mathfrak{A}_{\diamond}}^{\delta_{+}, \delta_{0}} \cong \mathfrak{I}_{\mathfrak{X}_{0}^{\dagger} / \mathfrak{C}_{0}^{\dagger}}^{\delta}
$$

in Lemma [5.2, we have an identification of virtual cycles

$$
\left[\mathfrak{I}_{\mathfrak{X}_{0}^{\dagger} / \mathfrak{C}_{0}^{\dagger}}^{\delta}\right]^{\text {vir }}=\left[\mathfrak{I}_{\mathfrak{X}_{+} / \mathfrak{A}_{\diamond}}^{\delta_{+}, \delta_{0}}\right]^{\text {vir }} \times\left[\mathfrak{I}_{\mathfrak{X}_{-} / \mathfrak{A}_{\diamond}}^{\delta_{-}, \delta_{0}}\right]^{\text {vir }}
$$

if we choose appropriate orientations on D-orbifolds associated with $\mathfrak{I}_{\mathfrak{X}_{ \pm} / \mathfrak{A}_{\diamond}}^{\delta_{ \pm}, \delta_{0}}$ and $\mathfrak{I}_{\mathfrak{X}_{0}^{\dagger} / \mathfrak{C}_{0}^{\dagger}}^{\delta}$ 
5.3. A conjectural gluing formula. We state a conjectural gluing formula of $D T_{4}$ invariants for a simple degeneration $\mathcal{X} \rightarrow C$ of projective $C Y_{4}$ 's. We assume $\omega_{\mathcal{X} / C}=0$ and $X_{0}=X_{+} \cup_{Y} X_{-}$with $Y$ as an anti-canonical divisor of $X_{ \pm}$.

We first consider the virtual cycle of $\mathfrak{I}_{\mathfrak{X} / \mathfrak{C}}^{P}$. Because of the triviality of the relative canonical bundle $\omega_{\mathcal{X} / C}=0$, as in Conjectures 5.8 and 5.9, there should exist a D-orbifold associated with $\mathfrak{I}_{\mathfrak{X} / \mathfrak{C}}^{P}$ and a Borel-Moore homology class $\left[\mathfrak{I}_{\mathfrak{X} / \mathfrak{C}}^{P}\right]^{\text {vir }} \in$ $H_{*}^{B M}\left(\mathfrak{I}_{\mathfrak{X} / \mathfrak{C}}^{P}, \mathbb{Q}\right)$ if the D-orbifold is orientable [30].

The comparison of $\left[\mathfrak{I}_{\mathfrak{X} / \mathfrak{C}}^{P}\right]^{\text {vir }}$ and $\left[\mathfrak{I}_{\mathfrak{X}_{0}^{\dagger} / \mathfrak{C}_{0}^{\dagger}}^{\delta}\right]^{\text {vir. By Lemma } 5.2} \mathfrak{I}_{\mathfrak{X}_{0}^{\dagger} / \mathfrak{C}_{0}^{\dagger}}^{\delta}$ is the zero locus of a section $\pi_{P}^{*} s_{\delta}$ of a complex line bundle $\pi_{P}^{*} L_{\delta}$ on $\mathfrak{I}_{\mathfrak{X} / \mathfrak{C}}^{P}$. Meanwhile, by [40], the obstruction theory of $\mathfrak{I}_{\mathfrak{X}_{0}^{\dagger} / \mathfrak{C}_{0}^{\dagger}}^{\delta}$ is the pull-back of the obstruction theory of $\mathfrak{I}_{\mathfrak{X} / \mathfrak{C}}^{P}$. Thus we should have

$$
\left[\mathfrak{I}_{\mathfrak{X}_{0}^{\dagger} / \mathfrak{C}_{0}^{\dagger}}\right]^{\text {vir }}=c_{1}\left(\pi_{P}^{*} L_{\delta}, \pi_{P}^{*} s_{\delta}\right) \cap\left[\mathfrak{I}_{\mathfrak{X} / \mathfrak{C}}^{P}\right]^{\text {vir }},
$$

where $c_{1}\left(\pi_{P}^{*} L_{\delta}, \pi_{P}^{*} s_{\delta}\right) \in H^{2}\left(\mathfrak{I}_{\mathfrak{X} / \mathfrak{C}}^{P}, \mathfrak{I}_{\mathfrak{X} / \mathfrak{C}}^{P}-\mathfrak{I}_{\mathfrak{X}_{0}^{\dagger} / \mathfrak{C}_{0}^{\dagger}}^{\delta}\right)$ is the localized first Chern class (Proposition 19.1.2, [23]) and $c_{1}\left(\pi_{P}^{*} L_{\delta}, \pi_{P}^{*} s_{\delta}\right) \cap: H_{*}^{B M}\left(\mathfrak{I}_{\mathfrak{X} / \mathfrak{C}}^{P}\right) \rightarrow H_{*-2}\left(\mathfrak{I}_{\mathfrak{X}_{0}^{\dagger} / \mathfrak{C}_{0}^{\dagger}}^{\delta}\right)$ is the cap product 29 .

Summing over all splitting $\delta$ of $P$, we get

$$
\sum_{\delta \in \Lambda_{P}^{s p l}}\left[\mathfrak{I}_{\mathfrak{X}_{0}^{\dagger} / \mathfrak{C}_{0}^{\dagger}}\right]^{\text {vir }}=c_{1}\left(\bigotimes_{\delta \in \Lambda_{P}^{s p l}} \pi_{P}^{*} L_{\delta}, \prod_{\delta \in \Lambda_{P}^{s p l}} \pi_{P}^{*} s_{\delta}\right) \cap\left[\mathfrak{I}_{\mathfrak{X} / \mathfrak{C}}^{P}\right]^{v i r}
$$

The comparison of $\left[\mathfrak{I}_{\mathfrak{X} / \mathfrak{C}}^{P}\right]^{\text {vir }}$ and $\left[\mathfrak{I}_{X_{t}}^{P}\right]^{\text {vir }}$. For $t \neq 0$, we have $\mathfrak{I}_{\mathfrak{X} / \mathfrak{C}}^{P} \times_{C} t \cong \mathfrak{I}_{X_{t}}^{P}$. The obstruction theory of $\mathfrak{I}_{X_{t}}^{P}$ is the pull-back of the obstruction theory of $\mathfrak{I}_{\mathfrak{X} / \mathfrak{C}}^{P}$. Without loss of generality, we assume $C=\mathbb{A}^{1}$ and similarly obtain

$$
\left[\mathfrak{I}_{X_{t}}^{P}\right]^{\text {vir }}=c_{1}\left(\mathcal{O}_{\mathfrak{I}_{\mathfrak{X} / \mathfrak{C}}^{P}}, \pi_{P}^{*} \pi^{*} t\right) \cap\left[\mathfrak{I}_{\mathfrak{X} / \mathfrak{C}}^{P}\right]^{\text {vir }},
$$

where $\left[\mathfrak{I}_{X_{t}}^{P}\right]^{\text {vir }}$ is the $D T_{4}$ virtual cycle mentioned in Theorem 2.6.

Conjecture 5.10. We take a simple degeneration $\mathcal{X} \rightarrow C$ of projective $C Y_{4}$ 's with $\omega_{\mathcal{X} / C}=0$ such that $X_{0}=X_{+} \cup_{Y} X_{-}$and $Y$ is an anti-canonical divisor of $X_{ \pm}$. Then there exist a D-orbifold associated with $\mathfrak{I}_{\mathfrak{X} / \mathfrak{C}}^{P}$ and a Borel-Moore homology class

$$
\left[\mathfrak{I}_{\mathfrak{X} / \mathfrak{C}}^{P}\right]^{\text {vir }} \in H_{*}^{B M}\left(\mathfrak{I}_{\mathfrak{X} / \mathfrak{C}}^{P}, \mathbb{Q}\right)
$$

if the corresponding D-orbifold is orientable.

Furthermore, equalities (11) and (13) hold if we choose appropriate orientations for D-orbifolds associated with $\mathfrak{I}_{\mathfrak{X} / \mathfrak{C}}^{P}, \mathfrak{I}_{\mathfrak{X}_{0}^{\dagger} / \mathfrak{C}_{0}^{\dagger}}^{\delta}$ and $\mathfrak{I}_{X_{t}}^{P}$.

To introduce the gluing formula, we make the following definition.

Definition 5.11. Let $\mathcal{X} \rightarrow C$ be a simple degeneration of projective $C Y_{4}$ 's such that $X_{0}=X_{+} \cup_{Y} X_{-}$with $Y$ as an anti-canonical divisor of $X_{ \pm} . P$ is a polynomial and $\mathfrak{I}_{\mathfrak{X} / \mathfrak{C}}^{P} \rightarrow C$ is Li-Wu's good degeneration of $\operatorname{Hilb}^{P}\left(X_{t}\right), t \neq 0$. We assume for any $\delta=\left(\delta_{ \pm}, \delta_{0}\right) \in \Lambda_{P}^{s p l}$, any closed point $I_{C} \in \operatorname{Hilb}^{\delta_{0}}(Y)$ satisfies $\operatorname{Ext}_{Y}^{1}\left(I_{C}, I_{C}\right)=0$. Then the family version $D T_{4}$ invariant of $\operatorname{Hilb}^{P}\left(X_{t}\right), t \neq 0$, is a map

$$
D T_{4}\left(\mathfrak{I}_{X_{t}}^{P}\right): \operatorname{Sym}^{*}\left(H_{*}(\mathcal{X}, \mathbb{Z}) \otimes \mathbb{Z}\left[x_{1}, x_{2}, \ldots\right]\right) \rightarrow \mathbb{Z}
$$


such that

$$
D T_{4}\left(\mathfrak{I}_{X_{t}}^{P}\right)\left(\left(\gamma_{1}, P_{1}\right),\left(\gamma_{2}, P_{2}\right), \ldots\right)=\int_{\left[\mathfrak{I}_{X_{t}}^{P}\right]^{\text {vir }}} \mu\left(\gamma_{1}, P_{1}\right) \cup \mu\left(\gamma_{2}, P_{2}\right) \cup \ldots,
$$

where $\gamma_{i} \in H_{*}(X, \mathbb{Z}), \mu($,$) is the \mu$-map defined in Definition 3.4 with respect to the universal sheaf of $\mathfrak{I}_{\mathfrak{X} / \mathfrak{C}}^{P}$, and we view $\mathfrak{I}_{X_{t}}^{P} \hookrightarrow \mathfrak{I}_{\mathfrak{X} / \mathfrak{C}}^{P}$ as a closed substack to do integration.

We take a Künneth type decomposition of the cohomology class

$$
\left.\left(\mu\left(\gamma_{1}, P_{1}\right) \cup \mu\left(\gamma_{2}, P_{2}\right) \cup \ldots\right)\right|_{\mathfrak{I}_{\mathfrak{X}_{0}^{\dagger} / \mathfrak{e}_{0}^{\dagger}}^{\dagger}}=\sum_{i} \tau_{+, \delta, i} \otimes \tau_{-, \delta, i} \in H^{*}\left(\mathfrak{I}_{\mathfrak{X}_{-} / \mathfrak{A}_{\diamond}}^{\delta_{-}, \delta_{0}} \times \mathfrak{I}_{\mathfrak{X}_{+} / \mathfrak{A}_{\diamond}}^{\delta_{+}, \delta_{0}}\right) .
$$

Then the relative $D T_{4}$ invariant of $\mathfrak{I}_{\mathfrak{X}_{ \pm} / \mathfrak{A}_{\diamond}}^{\delta_{ \pm}, \delta_{0}}$ with respect to $\tau_{ \pm, \delta, i}$ is

$$
D T_{4}\left(\mathfrak{I}_{\mathfrak{X}_{ \pm} / \delta_{\diamond}}^{\delta_{ \pm}, \delta_{0}}\right)\left(\tau_{ \pm, \delta, i}\right)=\int_{\left[\mathfrak{I}_{\mathfrak{X}_{ \pm} / \mathfrak{Q}_{\diamond}}^{\delta_{ \pm}, \delta_{0}}\right] \text { vir }} \tau_{ \pm, \delta, i} \in \mathbb{Q}
$$

We state a gluing formula of $D T_{4}$ invariants on Calabi-Yau 4-folds based on previous conjectures.

Theorem 5.12. Let $\mathcal{X} \rightarrow C$ be a simple degeneration of projective $C Y_{4}$ such that $\omega_{\mathcal{X} / C}=0$ and $X_{0}=X_{+} \cup_{Y} X_{-}$with $Y$ as an anti-canonical divisor of $X_{ \pm} . P$ is a polynomial and $\mathfrak{I}_{\mathfrak{X} / \mathfrak{C}}^{P} \rightarrow C$ is Li-Wu's good degeneration of $\operatorname{Hilb}^{P}\left(X_{t}\right), t \neq 0$. We assume for any $\delta=\left(\delta_{ \pm}, \delta_{0}\right) \in \Lambda_{P}^{\text {spl }}$, any closed point $I_{C} \in H_{i l b} \delta_{0}(Y)$ satisfies $\operatorname{Ext}_{Y}^{1}\left(I_{C}, I_{C}\right)=0$ (without loss of generality, we assume Hilb ${ }^{\delta_{0}}(Y)$ consists of one point for simplicity). Then for $t \neq 0 \in C$,

$D T_{4}\left(\mathfrak{I}_{X_{t}}^{P}\right)\left(\left(\gamma_{1}, P_{1}\right),\left(\gamma_{2}, P_{2}\right), \ldots\right)=\sum_{\delta \in \Lambda_{P}^{s p l}, i} D T_{4}\left(\mathfrak{I}_{\mathfrak{X}_{+} / \mathfrak{A}_{\diamond}}^{\delta_{+}, \delta_{0}}\right)\left(\tau_{+, \delta, i}\right) \cdot D T_{4}\left(\mathfrak{I}_{\mathfrak{X}_{-} / \mathfrak{A}_{\diamond}}^{\delta_{-}, \delta_{0}}\right)\left(\tau_{-, \delta, i}\right)$,

where $\gamma_{i} \in H_{*}(X, \mathbb{Z}), \mu($,$) is the \mu$-map defined in Definition 3.4 with respect to the universal sheaf of $\mathfrak{I}_{\mathfrak{X} / \mathfrak{C}}^{P},\left(\tau_{ \pm, \delta, i}\right)$ is the factor in a Künneth type decomposition as in Definition 5.11 .

Proof. By Lemma 5.2, Conjectures 5.9, 5.10 and (12).

Remark 5.13. By Corollary 2.16 of [57, if $P$ is the Hilbert polynomial associated with structure sheaves of points, then the condition which says for any $\delta=$ $\left(\delta_{ \pm}, \delta_{0}\right) \in \Lambda_{P}^{s p l}$ and any closed point $I_{C} \in \operatorname{Hilb}^{\delta_{0}}(Y)$, we have $\operatorname{Ext}_{Y}^{1}\left(I_{C}, I_{C}\right)=0$ is satisfied.

\section{Appendix on the orientability of Relative $D T_{4}$ Theory}

In this section, we give a coherent description of orientability issues involved in definitions of relative $D T_{4}$ virtual cycles (in section 3 ) and then give some partial verification for the existence of orientations.

We take a smooth (Calabi-Yau) 3 -fold $Y$ in a complex projective 4 -fold $X$ as its anti-canonical divisor, and denote $\mathfrak{M}_{X}$ to be a moduli space of stable bundles on $X$ with fixed Chern classes. Assuming conditions in Theorem 3.1 are satisfied, we obtain a morphism

$$
r: \mathfrak{M}_{X} \rightarrow \mathfrak{M}_{Y}
$$


to a Gieseker moduli space of stable sheaves on $Y$. We denote the determinant line bundle of $\mathfrak{M}_{X}$ by $\mathcal{L}_{X}$ with $\left.\mathcal{L}_{X}\right|_{E} \cong \operatorname{det}\left(H^{\text {odd }}(X, E n d E)\right) \otimes \operatorname{det}\left(H^{\text {even }}(X, E n d E)\right)^{-1}$ (similarly for $\mathcal{L}_{Y} \rightarrow \mathfrak{M}_{Y}$ ). In this set-up, there exists a canonical isomorphism

$$
\alpha:\left(\mathcal{L}_{\mathcal{M}_{X}}\right)^{\otimes 2} \cong r^{*} \mathcal{L}_{\mathcal{M}_{Y}} 6
$$

Definition 6.1. A relative orientation for morphism $r$ consists of a square root $\left(\left.\mathcal{L}_{\mathcal{M}_{Y}}\right|_{\mathcal{M}_{Y}^{\text {red }}}\right)^{\frac{1}{2}}$ of the determinant line bundle $\left.\mathcal{L}_{\mathcal{M}_{Y}}\right|_{\mathcal{M}_{Y}^{\text {red }}}$ and an isomorphism

$$
\theta:\left.\mathcal{L}_{\mathcal{M}_{X}}\right|_{\mathcal{M}_{X}^{\text {red }}} \cong r^{*}\left(\left.\mathcal{L}_{\mathcal{M}_{Y}}\right|_{\mathcal{M}_{Y}^{\text {red }}}\right)^{\frac{1}{2}}
$$

such that $\theta \otimes \theta \cong \alpha$ holds over $\mathcal{M}_{X}^{\text {red }}$ for the isomorphism $\alpha$.

Proposition 6.2. The restriction morphism having a relative orientation in Cases I-III individually is equivalent to the existence of an orientation in each corresponding case, i.e.

(i) the D-manifold associated with $\mathfrak{M}_{X}$ is orientable in Case I;

(ii) the self-dual obstruction bundle is orientable in Case II;

(iii) the self-dual reduced obstruction bundle is orientable in Case III.

Proof. As $\mathfrak{M}_{Y}$ is smooth in all cases, $\mathcal{L}_{Y}$ has a canonical square root given by $\operatorname{det}\left(T \mathfrak{M}_{Y}\right)$.

In Case I, $\mathfrak{M}_{Y}$ consists of a finite number of points. The existence of relative orientations is obviously equivalent to the existence of orientations for the D-manifold associated with $\mathfrak{M}_{X}$ (see also Theorem 2.7).

In Case II, $H^{*}(X, E n d E)$ and $H^{1}\left(Y,\left.E n d E\right|_{Y}\right)$ are locally constant. We abuse notation and use them also to denote the corresponding bundles. By the short exact sequence

$$
0 \rightarrow H^{3}(X, E n d E)^{*} \rightarrow H^{1}(X, E n d E) \rightarrow r^{*} H^{1}\left(Y,\left.E n d E\right|_{Y}\right) \rightarrow 0
$$

in Case II, the relative orientability is equivalent to the structure group of the obstruction bundle $H^{2}(X, E n d E)$ being reduced to $S O(\bullet, \mathbb{C})$; i.e. the self-dual obstruction bundle is orientable.

In Case III, the argument is similar to Case II.

We have the following partial verification of the existence of relative orientations.

Theorem 6.3 (Weak relative orientability). Let $Y$ be a smooth anti-canonical divisor in a projective 4-fold $X$ with $\operatorname{Tor}\left(H_{*}(X, \mathbb{Z})\right)=0$, and $E \rightarrow X$ be a complex vector bundle with structure group $S U(N)$, where $N \gg 0$. Let $\mathcal{M}_{X}$ be a coarse moduli scheme of simple holomorphic structures on $E$, which has a well-defined restriction morphism

$$
r: \mathcal{M}_{X} \rightarrow \mathcal{M}_{Y}
$$

to a proper coarse moduli scheme of simple bundles on $Y$ with fixed Chern classes.

Then there exists a square root $\left(\left.\mathcal{L}_{\mathcal{M}_{Y}}\right|_{\mathcal{M}_{Y}^{\text {red }}}\right)^{\frac{1}{2}}$ of $\left.\mathcal{L}_{\mathcal{M}_{Y}}\right|_{\mathcal{M}_{Y}^{\text {red }}}$ such that

$$
c_{1}\left(\left.\mathcal{L}_{\mathcal{M}_{X}}\right|_{\mathcal{M}_{X}^{\text {red }}}\right)=r^{*} c_{1}\left(\left(\left.\mathcal{L}_{\mathcal{M}_{Y}}\right|_{\mathcal{M}_{Y}^{\text {red }}}\right)^{\frac{1}{2}}\right),
$$

where $\mathcal{L}_{\mathcal{M}_{X}}$ (resp. $\mathcal{L}_{\mathcal{M}_{Y}}$ ) is the determinant line bundle of $\mathcal{M}_{X}$ (resp. $\mathcal{M}_{Y}$ ). Proof. See the proof of Theorem 4.1 of $[14$.

\footnotetext{
${ }^{6}$ See for instance Lemma 4.2 of [14].
} 
Another partial result is given as follows.

Proposition 6.4. We assume that $H^{1}\left(\mathcal{M}_{X}, \mathbb{Z}_{2}\right)=0$. Then relative orientations for restriction morphism $r: \mathcal{M}_{X} \rightarrow \mathcal{M}_{Y}$ exist.

Proof. See Proposition 4.6 of [14].

\section{ACKNOWLEDGEMENTS}

The first author is very grateful to Baosen $\mathrm{Wu}$ for many helpful discussions and Dominic Joyce for introducing him to his program of studying TQFT structures on Calabi-Yau 3- and 4-folds during a visit to the Simons Center for Geometry and Physics in May 2014. He also expresses his deep gratitude to Simon Donaldson for useful discussions and inviting him to give a talk on $D T_{4}$ theory in the G2 manifolds workshop at the Simons Center in September 2014, where part of this work was done. The authors thank Yan Soibelman for comments on the appropriate citation of references and are grateful to the referee for useful suggestions.

\section{REFERENCES}

[1] Michael Atiyah, New invariants of 3- and 4-dimensional manifolds, The mathematical heritage of Hermann Weyl (Durham, NC, 1987), Proc. Sympos. Pure Math., vol. 48, Amer. Math. Soc., Providence, RI, 1988, pp. 285-299, DOI 10.1090/pspum/048/974342. MR974342

[2] Michael Atiyah, Topological quantum field theories, Inst. Hautes Études Sci. Publ. Math. 68 (1988), 175-186 (1989). MR:1001453

[3] M. F. Atiyah and I. M. Singer, The index of elliptic operators. IV, Ann. of Math. (2) 93 (1971), 119-138, DOI 10.2307/1970756. MR0279833

[4] Kai Behrend, Donaldson-Thomas type invariants via microlocal geometry, Ann. of Math. (2) 170 (2009), no. 3, 1307-1338, DOI 10.4007/annals.2009.170.1307. MR2600874

[5] Kai Behrend, Jim Bryan, and Balázs Szendrői, Motivic degree zero Donaldson-Thomas invariants, Invent. Math. 192 (2013), no. 1, 111-160, DOI 10.1007/s00222-012-0408-1. MR3032328

[6] K. Behrend and B. Fantechi, The intrinsic normal cone, Invent. Math. 128 (1997), no. 1, 45-88, DOI 10.1007/s002220050136. MR 1437495

[7] D. Borisov and D. Joyce, Virtual fundamental classes for moduli spaces of sheaves on CalabiYau four-folds, arXiv:1504.00690, 2015.

[8] C. Brav, V. Bussi, D. Dupont, D. Joyce, and B. Szendrői, Symmetries and stabilization for sheaves of vanishing cycles, J. Singul. 11 (2015), 85-151. MR3353002

[9] C. Brav, V. Bussi, and D. Joyce, A 'Darboux theorem' for derived schemes with shifted symplectic structure, arXiv:1305.6302, 2013.

[10] Tom Bridgeland, Stability conditions on triangulated categories, Ann. of Math. (2) 166 (2007), no. 2, 317-345, DOI 10.4007/annals.2007.166.317. MR2373143

[11] Damien Calaque, Lagrangian structures on mapping stacks and semi-classical TFTs, Stacks and categories in geometry, topology, and algebra, Contemp. Math., vol. 643, Amer. Math. Soc., Providence, RI, 2015, pp. 1-23, DOI 10.1090/conm/643/12894. MR3381468

[12] Y. Cao, Donaldson-Thomas theory for Calabi-Yau four-folds, MPhil thesis, arXiv:1309.4230, 2013.

[13] Y. Cao and N. C. Leung, Donaldson-Thomas theory for Calabi-Yau 4-folds, arXiv:1407.7659, 2014.

[14] Y. Cao and N. C. Leung, Orientability for gauge theories on Calabi-Yau manifolds, arXiv:1502.01141, 2015.

[15] David A. Cox and Sheldon Katz, Mirror symmetry and algebraic geometry, Mathematical Surveys and Monographs, vol. 68, American Mathematical Society, Providence, RI, 1999. MR.1677117

[16] S. K. Donaldson, The orientation of Yang-Mills moduli spaces and 4-manifold topology, J. Differential Geom. 26 (1987), no. 3, 397-428. MR910015

[17] S. K. Donaldson, Infinite determinants, stable bundles and curvature, Duke Math. J. 54 (1987), no. 1, 231-247, DOI 10.1215/S0012-7094-87-05414-7. MR885784 
[18] S. K. Donaldson, Floer homology groups in Yang-Mills theory, Cambridge Tracts in Mathematics, vol. 147, Cambridge University Press, Cambridge, 2002. MR.1883043

[19] S. K. Donaldson and R. P. Thomas, Gauge theory in higher dimensions, The geometric universe (Oxford, 1996), Oxford Univ. Press, Oxford, 1998, pp. 31-47. MR.1634503

[20] Dan Edidin and William Graham, Characteristic classes and quadric bundles, Duke Math. J. 78 (1995), no. 2, 277-299, DOI 10.1215/S0012-7094-95-07812-0. MR1333501

[21] Hubert Flenner, Restrictions of semistable bundles on projective varieties, Comment. Math. Helv. 59 (1984), no. 4, 635-650, DOI 10.1007/BF02566370. MR780080

[22] Kenji Fukaya, Yong-Geun Oh, Hiroshi Ohta, and Kaoru Ono, Lagrangian intersection Floer theory: anomaly and obstruction. Part I, AMS/IP Studies in Advanced Mathematics, vol. 46, American Mathematical Society, Providence, RI; International Press, Somerville, MA, 2009. MR2553465

[23] William Fulton, Intersection theory, Ergebnisse der Mathematik und ihrer Grenzgebiete (3) [Results in Mathematics and Related Areas (3)], vol. 2, Springer-Verlag, Berlin, 1984. MR732620

[24] R. Gopakumar and C. Vafa, M-theory and topological strings II, arXiv:hep-th/9812127, 1998.

[25] H. Hofer, Polyfolds and Fredholm theory, arXiv:1412.4255, 2014.

[26] Shinobu Hosono, Masa-Hiko Saito, and Atsushi Takahashi, Relative Lefschetz action and BPS state counting, Internat. Math. Res. Notices 15 (2001), 783-816, DOI 10.1155/S107379280100040X. MR1849482

[27] Z. Hua, Orientation data on moduli space of sheaves on Calabi-Yau threefold, arXiv:1212.3790v4, 2015.

[28] Daniel Huybrechts and Richard P. Thomas, Deformation-obstruction theory for complexes via Atiyah and Kodaira-Spencer classes, Math. Ann. 346 (2010), no. 3, 545-569, DOI 10.1007/s00208-009-0397-6. MR2578562

[29] Birger Iversen, Cohomology of sheaves, Universitext, Springer-Verlag, Berlin, 1986. MR 842190

[30] D. Joyce, D-manifolds and d-orbifolds: a theory of derived differential geometry, book in preparation, 2012. Preliminary version available on Joyce's homepage.

[31] D. Joyce, A series of three talks given in Miami, January 2014, homepage of D. Joyce.

[32] Dominic Joyce and Yinan Song, A theory of generalized Donaldson-Thomas invariants, Mem. Amer. Math. Soc. 217 (2012), no. 1020, iv+199, DOI 10.1090/S0065-9266-2011-00630-1. MR.2951762

[33] Y. H. Kiem and J. Li, Categorification of Donaldson-Thomas invariants via perverse sheaves, arXiv:1212.6444v4, 2013.

[34] M. Kontsevich and Y. Soibelman, Stability structures, motivic Donaldson-Thomas invariants and cluster transformations, arXiv:0811.2435, 2008.

[35] Peter Kronheimer and Tomasz Mrowka, Monopoles and three-manifolds, New Mathematical Monographs, vol. 10, Cambridge University Press, Cambridge, 2007. MR2388043

[36] Naichung Conan Leung, Topological quantum field theory for Calabi-Yau threefolds and $G_{2}$-manifolds, Adv. Theor. Math. Phys. 6 (2002), no. 3, 575-591, DOI 10.4310/ATMP.2002.v6.n3.a5. MR.1957671

[37] Jun Li, Stable morphisms to singular schemes and relative stable morphisms, J. Differential Geom. 57 (2001), no. 3, 509-578. MR 1882667

[38] Jun Li, A degeneration formula of $G W$-invariants, J. Differential Geom. 60 (2002), no. 2, 199-293. MR.1938113

[39] Jun Li and Gang Tian, Virtual moduli cycles and Gromov-Witten invariants of algebraic varieties, J. Amer. Math. Soc. 11 (1998), no. 1, 119-174, DOI 10.1090/S0894-0347-98-002501. MR 1467172

[40] Jun Li and Baosen Wu, Good degeneration of Quot-schemes and coherent systems, Comm. Anal. Geom. 23 (2015), no. 4, 841-921, DOI 10.4310/CAG.2015.v23.n4.a5. MR3385781

[41] Wei-Ping Li and Zhenbo Qin, Stable rank-2 bundles on Calabi-Yau manifolds, Internat. J. Math. 14 (2003), no. 10, 1097-1120, DOI 10.1142/S0129167X03002150. MR2031186

[42] Max Lieblich, Moduli of complexes on a proper morphism, J. Algebraic Geom. 15 (2006), no. 1, 175-206, DOI 10.1090/S1056-3911-05-00418-2. MR2177199

[43] D. Maulik, N. Nekrasov, A. Okounkov, and R. Pandharipande, Gromov-Witten theory and Donaldson-Thomas theory. I, Compos. Math. 142 (2006), no. 5, 1263-1285, DOI 10.1112/S0010437X06002302. MR2264664 
[44] D. Maulik, N. Nekrasov, A. Okounkov, and R. Pandharipande, Gromov-Witten theory and Donaldson-Thomas theory. II, Compos. Math. 142 (2006), no. 5, 1286-1304, DOI 10.1112/S0010437X06002314. MR2264665

[45] D. Maulik, A. Oblomkov, A. Okounkov, and R. Pandharipande, Gromov-Witten/DonaldsonThomas correspondence for toric 3-folds, Invent. Math. 186 (2011), no. 2, 435-479, DOI 10.1007/s00222-011-0322-y. MR2845622

[46] D. Maulik, R. Pandharipande, and R. P. Thomas, Curves on K3 surfaces and modular forms, J. Topol. 3 (2010), no. 4, 937-996, DOI 10.1112/jtopol/jtq030. MR2746343

[47] John McCleary, User's guide to spectral sequences, Mathematics Lecture Series, vol. 12, Publish or Perish, Inc., Wilmington, DE, 1985. MR820463

[48] Shigeru Mukai, Symplectic structure of the moduli space of sheaves on an abelian or K3 surface, Invent. Math. 77 (1984), no. 1, 101-116, DOI 10.1007/BF01389137. MR751133

[49] Nikita Nekrasov and Andrei Okounkov, Membranes and sheaves, Algebr. Geom. 3 (2016), no. 3, 320-369, DOI 10.14231/AG-2016-015. MR3504535

[50] R. Pandharipande and A. Pixton, Gromov-Witten/Pairs correspondence for the quintic 3fold, J. Amer. Math. Soc. 30 (2017), no. 2, 389-449, DOI 10.1090/jams/858. MR3600040

[51] R. Pandharipande and R. P. Thomas, Curve counting via stable pairs in the derived category, Invent. Math. 178 (2009), no. 2, 407-447, DOI 10.1007/s00222-009-0203-9. MR2545686

[52] Tony Pantev, Bertrand Toën, Michel Vaquié, and Gabriele Vezzosi, Shifted symplectic structures, Publ. Math. Inst. Hautes Études Sci. 117 (2013), 271-328, DOI 10.1007/s10240-0130054-1. MR3090262

[53] Paul Seidel and Richard Thomas, Braid group actions on derived categories of coherent sheaves, Duke Math. J. 108 (2001), no. 1, 37-108, DOI 10.1215/S0012-7094-01-10812-0. MR.1831820

[54] R. P. Thomas, A holomorphic Casson invariant for Calabi-Yau 3-folds, and bundles on K3 fibrations, J. Differential Geom. 54 (2000), no. 2, 367-438. MR.1818182

[55] Yukinobu Toda, Curve counting theories via stable objects I. DT/PT correspondence, J. Amer. Math. Soc. 23 (2010), no. 4, 1119-1157, DOI 10.1090/S0894-0347-10-00670-3. MR2669709

[56] K. Uhlenbeck and S.-T. Yau, On the existence of Hermitian-Yang-Mills connections in stable vector bundles, Comm. Pure Appl. Math. 39 (1986), no. S, suppl., S257-S293, DOI 10.1002/cpa.3160390714. Frontiers of the mathematical sciences: 1985 (New York, 1985). MR.861491

[57] B. Wu, The moduli stack of stable relative ideal sheaves, arXiv:math/0701074v1, 2007.

[58] Dingyu Yang, A Choice-independent Theory of Kuranishi Structures and the PolyfoldKuranishi Correspondence, ProQuest LLC, Ann Arbor, MI, 2014. Thesis (Ph.D.)-New York University. MR 3218117

[59] Shing Tung Yau, On the Ricci curvature of a compact Kähler manifold and the complex Monge-Ampère equation. I, Comm. Pure Appl. Math. 31 (1978), no. 3, 339-411, DOI 10.1002/cpa.3160310304. MR 480350

The Institute of Mathematical Sciences and Department of Mathematics, The ChiNese University of Hong Kong, Shatin, Hong Kong

E-mail address: ylcao@math.cuhk.edu.hk

The Institute of Mathematical Sciences and Department of Mathematics, The ChiNese University of Hong Kong, Shatin, Hong Kong

E-mail address: leung@math.cuhk.edu.hk 